\title{
MineRAL INCLUSIONS IN RUBY AND Sapphire from the Bo Welu GeM Deposit in Chanthaburi, Thailand
}

Supparat Promwongnan and Chakkaphan Sutthirat

The mineral inclusions of alluvial ruby and sapphire from the Bo Welu gem deposit in Thailand's Chanthaburi Province were collected and investigated. This deposit is directly related to a basaltic terrain. Raman spectroscopy and electron probe micro-analyzer (EPMA) measurements allow the identification of mineral inclusions of pyrope-rich garnet, sillimanite, high-Al diopside, sapphirine, nepheline, quartz, feldspar (mostly plagioclase), spinel, sulfide, anhydrite, and silicate melts in ruby and purple sapphire. Zircon, alkali feldspar (mostly with high Na content), monazite, columbite, and sulfide were identified in blue sapphire. This study represents the first report of several inclusions in ruby and sapphire from this gem deposit.

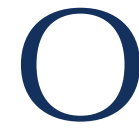
ccurrences of gem ruby and sapphire in Thailand are located in the provinces of Chanthaburi-Trat, Kanchanaburi, PhraeSukhothai, Ubon Ratchathani, Si Sa Ket, and Phetchabun (figure 1). However, a few main areas have been economically mined and supplied gems to the world market. Sapphire mines were once extensively operated in Kanchanaburi, but most have recently closed down. The Chanthaburi-Trat area has been the most significant source of Thai corundum, and a few small mines are still in operation there. The most famous source of Thai rubies is located in the Bo Rai deposit in the eastern part of Trat Province, close to the Cambodian province of Pailin (figure 1). What became known as "Siamese ruby" was first named from here and adjacent areas. Meanwhile, the main gem mining area for sapphire (yellow, green, and blue, along with golden star and black star sapphires) is the Bang $\mathrm{Ka}$ Cha deposit in western Chanthaburi (again, see figure 1). Most gem mines in the central deposits, around Khlung District within Chanthaburi Province, have supplied more sapphire than ruby (Vichit, 1992; Pattamalai, 2015).

The focus of this study, the Bo Welu gem field located in the central gem deposits, has been mined for

See end of article for About the Authors and Acknowledgments.

Gems \& Gemology, Vol. 55, No. 3, pp. 354-369,

http://dx.doi.org/10.5741/GEMS.55.3.354

(C) 2019 Gemological Institute of America both ruby and sapphire. Corundum from this area is generally heat-treated to enhance its color, especially the red (or purplish red) ruby and the blue sapphire (figure 2), which are in high demand, particularly within Thailand.

\section{In Brief}

- Various mineral inclusions are observed in Bo Welu ruby and sapphire. Although some of these minerals have already been reported as inclusions in Thai corundum, others are first reported here.

- Among the observations were nepheline, sillimanite, spinel, alkali feldspar, quartz, and anhydrite inclusions in ruby and purple sapphire, and columbite and sulfide inclusions in blue sapphire.

- Common mineral inclusions and their chemical compositions indicate different original formations of ruby and sapphire that appear to have the same thermal history of basaltic activity in this region.

Although gem mines in this area are not currently in operation, a few artisanal miners can be found working along streams during the rainy season. The rarity of Thai ruby ensures strong demand in the local and international markets and high value. The sapphires, meanwhile, possess a natural greenish blue color that becomes an intense blue after heating.

In this study, ruby and sapphire from Bo Welu were investigated to determine their mineral inclu- 


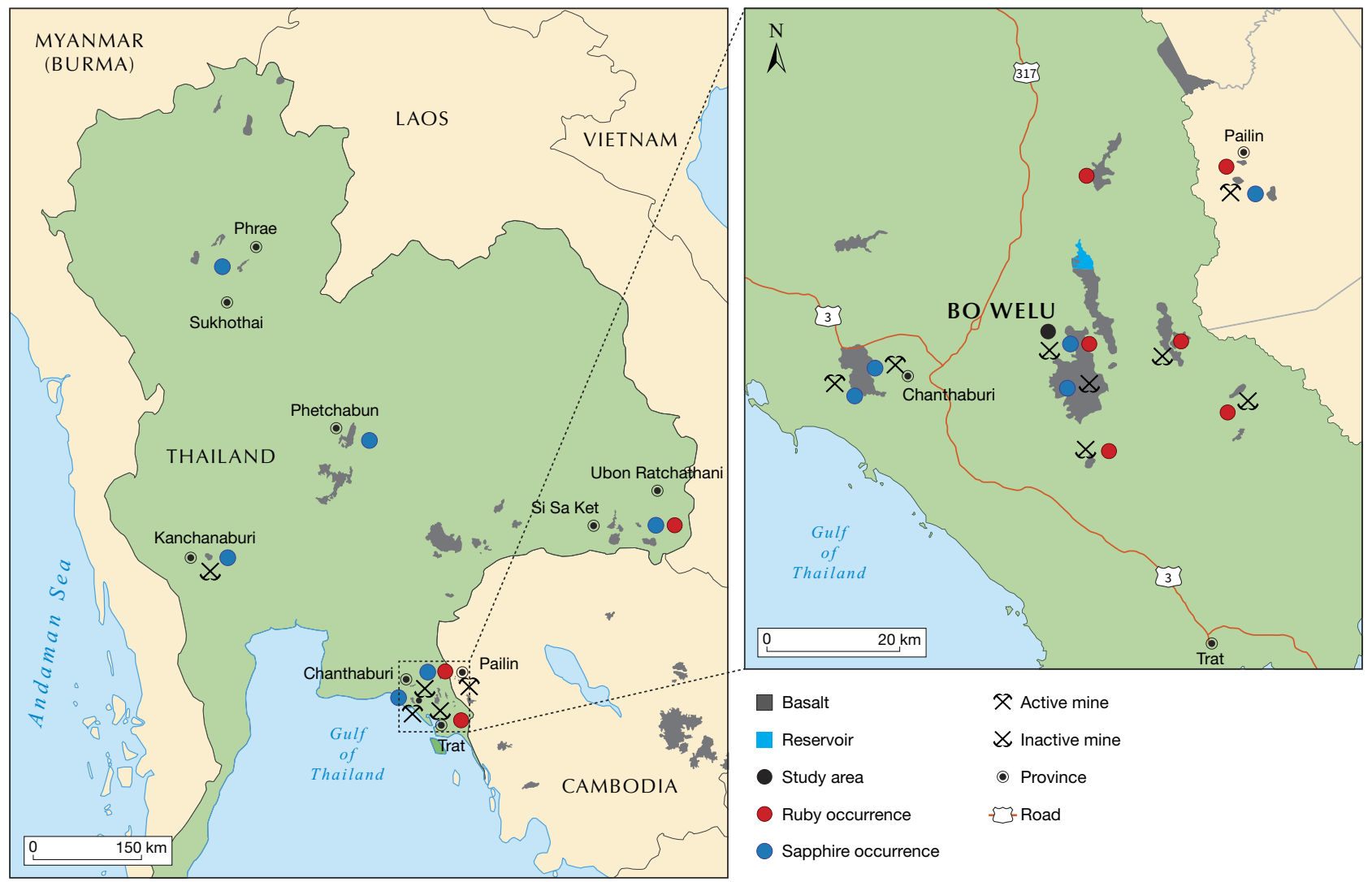

Figure 1. Left: Map of Thailand showing the distribution of basalt and corundum occurrences. Right: Ruby and sapphire deposits and associated basalts in Chanthaburi-Trat Province in eastern Thailand and Pailin Province in western Cambodia. Modified after Hughes (1997), Sutthirat et al. (2001), Barr and Cooper (2013), and Pattamalai (2015).

sions and compared with other Thai corundum and basaltic corundum from other deposits. In this study, rubies and purple sapphires are considered to-

Figure 2. Bo Welu ruby and sapphires set in rings. The intense blue sapphires are nearly $6 \mathrm{ct}$ (left) and $8 \mathrm{ct}$ (right). The deep red ruby in the middle is approximately $1 \mathrm{ct}$. These stones were heated to improve their color. Photo by Supparat Promwongnan, courtesy of Kannikar Rojvanakarn.

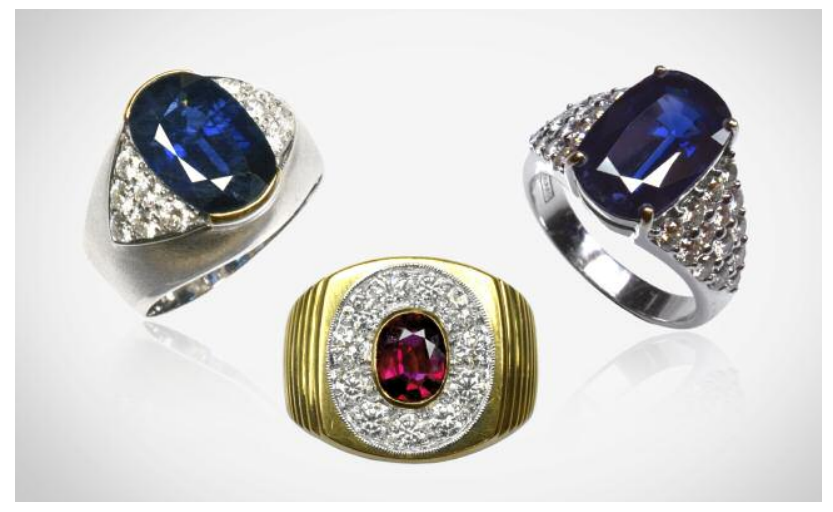

gether as a single genetic group that is presented in contrast to the blue sapphires, which are presumed have a different geologic origin. The results of this study may improve the understanding of genetic models of formation of basaltic ruby and sapphire in Thailand.

\section{MATERIALS AND METHODS}

More than 1,000 corundum grains from Bo Welu were collected from local miners nearly 20 years ago. The samples were examined under an optical microscope to search for mineral inclusions before the selected stones were carefully polished as slabs by a Facetron faceting machine to expose the mineral inclusions. A total of 99 corundum samples containing 191 tiny mineral inclusions were then selected for further investigation.

General properties such as size, color, specific gravity (SG), and refractive indices (RI) were measured using standard gemological equipment. External and internal features were observed under a gemological microscope. 


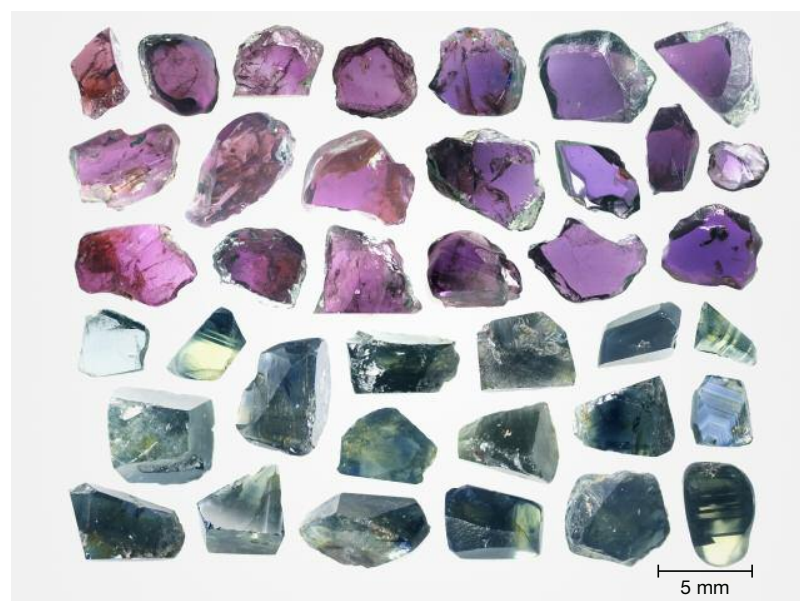

Figure 3. These representative ruby and sapphire samples collected from Bo Welu were polished for analyses in the study. Photo by $S$. Promwongnan.

The selected samples, after pre-screening of mineral inclusions by Raman spectroscopy, were polished and mounted in epoxy resin prior to carbon coating. The JEOL JXA-8100 electron probe microanalyzer (EPMA) at the geology department of Chulalongkorn University was used to analyze major and minor compositions. Operating conditions were set at $15 \mathrm{kV}$ accelerating voltage and about $25 \mathrm{nA}$ sample current in the focused beam (smaller than $1 \mu \mathrm{m}$ ). Measurement times for each element were set at 30 seconds for peak counts and 10 seconds for background counts. For special analyses of zircon and monazite inclusions, an accelerating voltage of $20 \mathrm{kV}$ was set to achieve more accurate results. Appropriate standard reference materials, including natural minerals, pure oxides, and pure elements, were used for calibration. Detection limits may reach 100-300 ppm under these conditions. An automatic ZAF cor- rection was applied to the analytical results before reporting percent oxides. $\mathrm{Fe}^{2+}$ and $\mathrm{Fe}^{3+}$ ratios of minerals such as garnet, pyroxene, sapphirine, and spinel were recalculated using the method of Droop (1987).

\section{RESULTS}

General Characteristics. Most of the ruby and purple sapphire samples were less than $0.7 \mathrm{ct}$ and measured up to $6 \mathrm{~mm}$ in length. Colors included purplish red, purple-red, reddish purple, and purple. The crystals generally displayed normal tabular habits with slightly rounded edges (figure 3). They were doubly refractive, with an RI of 1.760 to 1.770 and a birefringence of 0.009 to 0.010 . Most of the samples displayed weak to moderate red fluorescence under long-wave UV radiation and were inert under short-wave UV.

The blue sapphire samples ranged from 0.2 to 6.9 ct and measured up to $1 \mathrm{~cm}$ long. These crystals were mostly characterized by a hexagonal habit with parting along basal planes. Some stones without crystal faces (figure 3) were rounded due to corrosion in the carrier magma (see Coenraads, 1992). They had semi-transparent to transparent blue to greenish blue and greenblue colors with medium to dark tones. They had an RI ranging from 1.760 to 1.771 , with a corresponding birefringence of 0.009 to 0.010 . These samples were generally inert under long-wave and short-wave UV. Moreover, these rough rubies and sapphires sometimes presented a primary corroded surface (figure 4) caused by hot magma, which is typically observed in corundum from basaltic terrain (Coenraads, 1992).

Mineral Inclusions in Ruby and Purple Sapphire. Various microscopic features were observed in Bo Welu ruby and purple sapphire, such as healed fractures or

Figure 4. Photomicrographs of rough samples of ruby and sapphire, taken under reflected light. Left: Etched features on a ruby's surface (field of view $2.70 \mathrm{~mm}$ ). Center: Triangular etch marks on a ruby's surface (field of view $1.30 \mathrm{~mm}$ ). Right: Dissolved features on a sapphire's surface (field of view $3.78 \mathrm{~mm}$ ). Photomicrographs by $S$. Promwongnan.
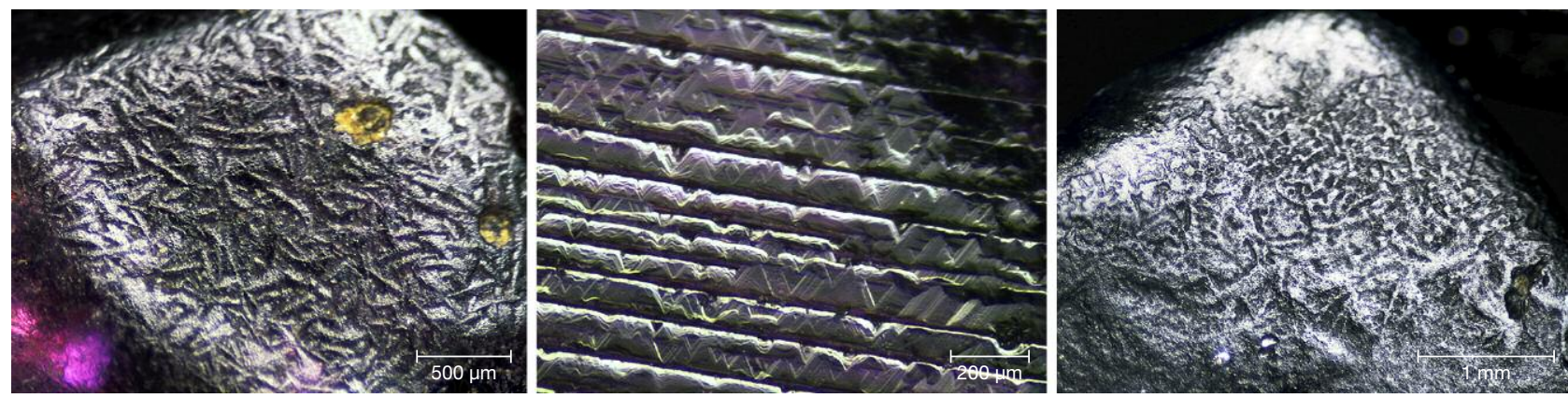

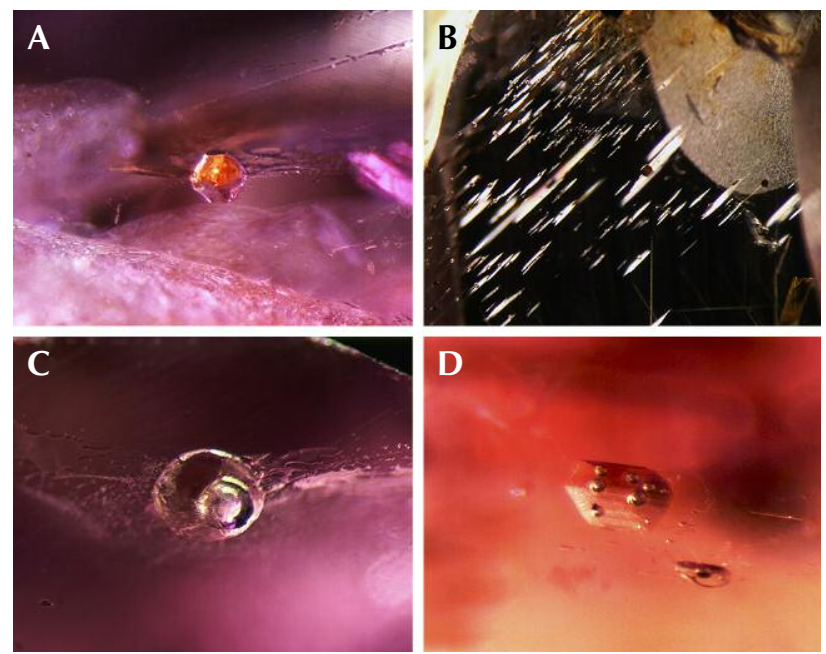

Figure 5. Some internal features observed in Bo Welu ruby and purple sapphire. A: Euhedral crystal surrounded by tension cracks (field of view $1.1 \mathrm{~mm}$ ). $B$ : Tiny crystals surrounded by thin films (field of view 3.0 $\mathrm{mm}$ ). $C$ and D: Two-phase inclusions (fields of view 1.4 $\mathrm{mm}$ and $0.7 \mathrm{~mm}$ ). Photomicrographs by $S$. Promwongnan; darkfield illumination with fiber-optic light.

fingerprints, networks of tiny crystals, euhedral crystals surrounded by tension cracks (figure 5A), and tiny crystal inclusions with equatorial thin films (figure 5B). The last is a typical feature observed in ruby from Thailand. Many two-phase inclusions were captured using a combination of fiber-optic and darkfield illumination (figure 5, C and D). Previous researchers proposed the presence of various mineral inclusions in ruby from Thailand as well as from Pailin, Cambodia. A few analytical techniques were engaged to identify these mineral inclusions, such as optical microscopy and Raman spectroscopy (e.g., Gübelin and Koivula, 1986; Hughes, 1997; Intasopa et al., 1999; Khamloet et al., 2014; Saeseaw et al., 2017; Sangsawong et al., 2017) and X-ray powder diffraction (XRD) (Koivula and Fryer, 1987). Moreover, mineral inclusion chemistries were also reported using EPMA (Gübelin, 1971; Guo et al., 1994; Sutherland et al., 1998a; Sutthirat et al., 2001; Khamloet et al., 2014), proton microprobe analysis (PMP) (Guo et al., 1994), and scanning electron microscopy with energy dispersive X-ray spectrometry (SEM-EDX) (Saminpanya and Sutherland, 2011).

For this study, exposed mineral inclusions were initially identified using Raman spectroscopy before their chemical compositions were analyzed by EPMA. It is noteworthy that a number of mineral inclusions in Thai ruby and purple sapphire are first re-
TABLE 1. Summary of mineral inclusions discovered in Bo Welu ruby and sapphire, compared to those reported from surrounding basaltic gem fields.

\begin{tabular}{|c|c|c|c|c|c|c|c|c|c|c|}
\hline & & \multicolumn{4}{|c|}{ Ruby, Purple Sapphire } & \multicolumn{5}{|c|}{ Blue Sapphire } \\
\hline $\begin{array}{l}\text { Mineral } \\
\text { group }\end{array}$ & $\begin{array}{l}\text { Mineral } \\
\text { inclusions }\end{array}$ & 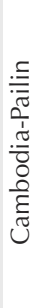 & 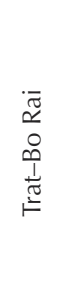 & $\begin{array}{l}\infty \\
0 \\
0 \\
3 \\
\frac{\pi}{Z} \\
\frac{1}{\pi} \\
\models\end{array}$ & 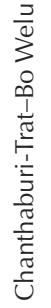 & 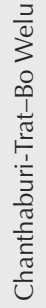 & 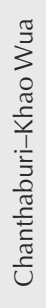 & 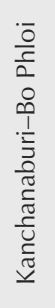 & 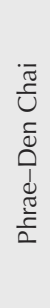 & 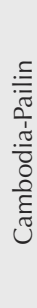 \\
\hline \multirow[t]{11}{*}{ Silicates } & Zircon & - & - & - & - & $\checkmark *$ & $\sqrt{ } 6$ & $\sqrt{ } 3,4$ & - & - \\
\hline & Garnet & - & $\sqrt{ } 2,3,9$ & - & $\checkmark *$ & - & - & $\sqrt{4}$ & - & - \\
\hline & Sillimanite & - & $\sqrt{ }^{9}$ & - & $\checkmark *$ & - & - & - & - & - \\
\hline & Staurolite & - & - & - & - & & - & $\checkmark^{4}$ & - & - \\
\hline & Diopside & $\sqrt{ }^{7}$ & $\sqrt{ } 2,3,9$ & - & $\checkmark *$ & - & - & - & - & - \\
\hline & Enstatite & - & - & - & - & - & - & $\sqrt{ }^{4}$ & - & - \\
\hline & Sapphirine & $\sqrt{ }^{7}$ & $\sqrt{5}$ & $\sqrt{ } 2,3$ & $\checkmark *$ & - & - & $\checkmark^{4}$ & - & - \\
\hline & Nepheline & - & - & - & $\checkmark *$ & - & - & $\sqrt{ } 3,4$ & - & - \\
\hline & Quartz & - & - & - & $\checkmark *$ & - & - & - & - & - \\
\hline & $\begin{array}{l}\text { Alkali } \\
\text { feldspar }\end{array}$ & - & - & - & $\checkmark *$ & $\checkmark *$ & - & $\sqrt{ }^{3,4}$ & $\sqrt{3}^{3}$ & - \\
\hline & Plagioclase & - & $\sqrt{ } 8,9$ & - & $\checkmark *$ & - & - & - & - & - \\
\hline \multirow[t]{2}{*}{ Oxides } & Spinel & $\sqrt{ }^{7}$ & $\sqrt{ }^{9}$ & - & $\checkmark *$ & - & - & $\checkmark^{1,3}$ & - & $\sqrt{7}^{7}$ \\
\hline & Ilmenite & - & - & - & - & - & - & $\sqrt{4}^{4}$ & - & - \\
\hline Niobium & Columbite & - & - & - & - & $\checkmark *$ & - & - & - & - \\
\hline Phosphates & Monazite & - & - & - & - & $\checkmark *$ & - & $\checkmark^{4}$ & - & - \\
\hline \multirow[t]{2}{*}{ Carbonates } & Calcite & - & - & - & - & - & - & $\checkmark^{4}$ & - & - \\
\hline & Sulfide & - & $\checkmark^{8,9}$ & - & $\checkmark *$ & $\checkmark *$ & - & - & - & - \\
\hline Sulfates & Anhydrite & - & - & - & $\checkmark *$ & - & - & - & - & - \\
\hline
\end{tabular}

*This study, using Raman and EPMA

'Guo et al. (1994), using EPMA and PMP

${ }^{2}$ Sutthirat et al. (2001), using EPMA

${ }^{3}$ Saminpanya and Sutherland (2011), using SEM-EDS

${ }^{4}$ Khamloet et al. (2014), using Raman and EPMA

${ }^{5}$ Koivula and Fryer (1987), using XRD

${ }^{6}$ Sutherland et al. (1998a), using EPMA

${ }^{7}$ Sutherland et al. (1998b), using EPMA

${ }^{8}$ Gübelin (1971), using EPMA

${ }^{9}$ Promwongnan and Sutthirat (2019), using Raman and EPMA

ported in this study (table 1). For comparison, mineral inclusions found in rubies from nearby mining areas in Chanthaburi-Trat and Pailin are summarized in the same table. Chemical analyses of these inclusions are also provided for further discussion.

Garnet with very low chromium content is often found in Bo Welu ruby and purple sapphire. The crystals usually occur as ellipsoidal, irregular shapes with poorly formed crystal faces (figure 6A) that are either colorless or pale purplish red. They have a pyrope-rich 
TABLE 2. EPMA analyses of garnet, sillimanite, pyroxene, sapphirine, nepheline, and quartz inclusions in Bo Welu ruby and purple sapphire samples.

\begin{tabular}{|c|c|c|c|c|c|c|c|c|c|}
\hline \multirow{2}{*}{$\begin{array}{l}\text { Mineral } \\
\text { phase } \\
\text { analysis } \\
\text { (wt.\%) }\end{array}$} & \multicolumn{2}{|c|}{ Garnet } & \multirow{2}{*}{$\begin{array}{c}\text { Sillimanite } \\
\text { rP } \\
\text { 9TWL152-1 }\end{array}$} & \multicolumn{2}{|c|}{ Diopside } & \multirow{2}{*}{$\begin{array}{l}\text { Sapphirine } \\
\text { PR } \\
\text { 9TWL160-1 }\end{array}$} & \multicolumn{2}{|c|}{ Nepheline } & \multirow{2}{*}{$\begin{array}{c}\text { Quartz } \\
\text { PR } \\
\text { 9TWL095-2 }\end{array}$} \\
\hline & $\begin{array}{c}\text { PR } \\
\text { 9TWL059-1 }\end{array}$ & $\begin{array}{c}\mathrm{P} \\
\text { 9TWL092-2 }\end{array}$ & & $\begin{array}{c}\text { PR } \\
\text { 9TWL029-1 }\end{array}$ & $\begin{array}{c}\text { rP } \\
\text { 9TWL035-1 }\end{array}$ & & $\begin{array}{c}\text { rP } \\
\text { 9TWL012-1 }\end{array}$ & $\begin{array}{c}\text { rP } \\
\text { 9TWL153-1 }\end{array}$ & \\
\hline $\mathrm{SiO}_{2}$ & 42.03 & 42.57 & 36.38 & 44.85 & 49.28 & 13.97 & 47.61 & 45.99 & 99.61 \\
\hline $\mathrm{TiO}_{2}$ & 0.07 & 0.10 & 0.01 & 0.24 & 0.75 & nd & nd & 0.07 & nd \\
\hline $\mathrm{Al}_{2} \mathrm{O}_{3}$ & 23.26 & 23.17 & 61.62 & 16.5 & 8.82 & 61.06 & 30.79 & 34.02 & 0.05 \\
\hline $\mathrm{Cr}_{2} \mathrm{O}_{3}$ & 0.02 & 0.05 & nd & 0.17 & 0.13 & 0.31 & nd & nd & nd \\
\hline $\mathrm{FeO}$ & 9.71 & 10.33 & 1.43 & 3.04 & 3.28 & 3.32 & 0.22 & 0.15 & 0.28 \\
\hline $\mathrm{MnO}$ & 0.20 & 0.19 & nd & nd & 0.01 & 0.03 & nd & nd & nd \\
\hline $\mathrm{MgO}$ & 15.82 & 15.21 & 0.50 & 11.41 & 12.88 & 19.62 & 0.20 & 0.05 & nd \\
\hline $\mathrm{NiO}$ & na & na & na & na & na & 0.41 & na & na & na \\
\hline $\mathrm{CaO}$ & 8.85 & 8.17 & 0.15 & 22.52 & 23.99 & 0.14 & 4.67 & 3.09 & 0.01 \\
\hline $\mathrm{K}_{2} \mathrm{O}$ & 0.01 & 0.02 & 0.01 & 0.02 & nd & 0.02 & 15.10 & 14.80 & nd \\
\hline $\mathrm{Na}_{2} \mathrm{O}$ & nd & 0.02 & nd & 1.11 & 0.76 & nd & 1.32 & 1.84 & nd \\
\hline Total & 99.98 & 99.82 & 100.10 & 99.85 & 99.90 & 98.87 & 99.91 & 100.01 & 99.95 \\
\hline Formula & \multicolumn{2}{|c|}{$12(\mathrm{O})$} & $20(\mathrm{O})$ & \multicolumn{2}{|c|}{$6(O)$} & $20(O)$ & \multicolumn{2}{|c|}{$4(O)$} & $2(O)$ \\
\hline $\mathrm{Si}$ & 3.026 & 3.026 & 3.958 & 1.639 & 1.805 & 1.655 & 1.116 & 1.074 & 0.998 \\
\hline $\mathrm{Ti}$ & 0.004 & 0.004 & 0.001 & 0.006 & 0.021 & 0.000 & 0.000 & 0.001 & 0.000 \\
\hline $\mathrm{Al}$ & 1.974 & 1.974 & 7.902 & 0.711 & 0.381 & 8.527 & 0.851 & 0.936 & 0.001 \\
\hline $\mathrm{Cr}$ & 0.001 & 0.001 & 0.000 & 0.005 & 0.004 & 0.029 & 0.000 & 0.000 & 0.000 \\
\hline${ }^{\mathrm{a}} \mathrm{Fe}^{3+}$ & 0.000 & 0.000 & 0.130 & 0.11 & 0.027 & 0.196 & 0.000 & 0.000 & 0.002 \\
\hline${ }^{\mathrm{a}} \mathrm{Fe}^{2+}$ & 0.584 & 0.584 & 0.000 & 0.000 & 0.073 & 0.133 & 0.004 & 0.003 & 0.000 \\
\hline $\mathrm{Mn}$ & 0.012 & 0.012 & 0.000 & 0.000 & 0.000 & 0.003 & 0.000 & 0.000 & 0.000 \\
\hline $\mathrm{Ca}$ & 0.683 & 0.683 & 0.017 & 0.882 & 0.942 & 0.017 & 0.117 & 0.077 & 0.000 \\
\hline K & 0.001 & 0.001 & 0.002 & 0.001 & 0.000 & 0.003 & 0.686 & 0.670 & 0.000 \\
\hline $\mathrm{Na}$ & 0.000 & 0.000 & 0.000 & 0.079 & 0.054 & 0.000 & 0.039 & 0.055 & 0.000 \\
\hline Total & 7.983 & 7.983 & 12.091 & 4.037 & 4.009 & 14.069 & 2.821 & 2.819 & 1.001 \\
\hline \multicolumn{10}{|c|}{$\begin{array}{l}{ }^{a} \mathrm{Fe}^{2+} \text { and } \mathrm{Fe}^{3+} \text { were recalculated from total } \mathrm{FeO} \text { at } \\
n d=\text { not detected } \\
\text { na }=\text { not analyzed } \\
P R=\text { purple-red }, r P=\text { reddish purple, } P=\text { purple }\end{array}$} \\
\hline
\end{tabular}

composition (high $\mathrm{Mg}$; see table 2), which is similar to garnet inclusions in Bo Rai ruby and purple sapphire located about $30 \mathrm{~km}$ southeast of the study area, previously reported by Sutthirat et al. (2001), Saminpanya and Sutherland (2011), and Promwongnan and Sutthirat (2019).

Sillimanite occurred rarely in this study, displaying a subhedral shape with a hexagonal habit surrounded by healed fractures (figure 6B). These inclusions contained major contents of $\mathrm{SiO}_{2}$ and $\mathrm{Al}_{2} \mathrm{O}_{3}$ and traces of $\mathrm{FeO}$ and $\mathrm{MgO}$ (again, see table 2). Sillimanite inclusions have recently been reported in ruby and purple sapphire from the Bo Rai gem field
(Promwongnan and Sutthirat, 2019). Therefore, these two studies represent the first discovery of sillimanite inclusions in Thai ruby and purple sapphire.

High-alumina diopside was the most common inclusion found in Bo Welu ruby and purple sapphire. They usually formed colorless rounded or ellipsoidal crystals (figure 6C), with twinning lamella sometimes observed. These inclusions were composed of an $\mathrm{Al}$ rich component (0.38-0.71 Al) with slightly higher $\mathrm{Ca}$ and lower $\mathrm{Mg}$ components (see table 2). Diopside inclusions were previously reported in alluvial ruby and purple sapphire from Bo Rai (e.g., Sutthirat et al., 2001; Saminpanya and Sutherland, 2011; Promwong- 

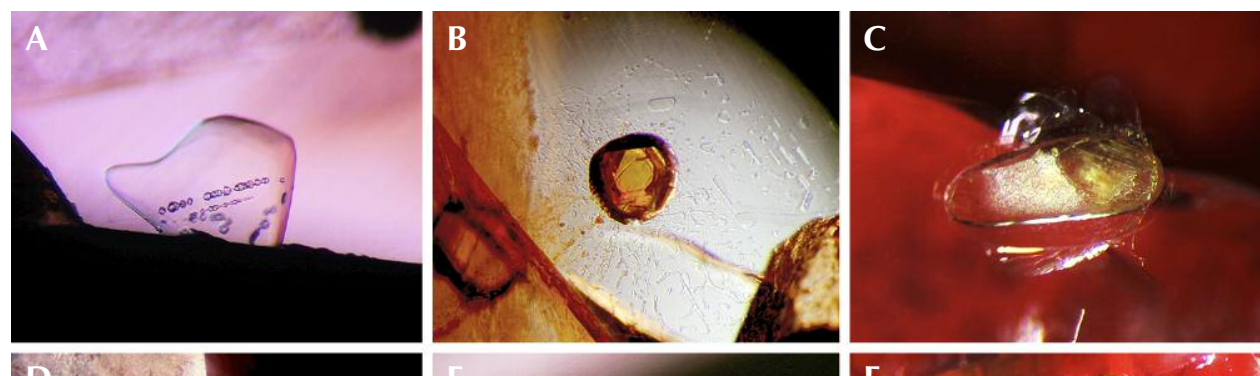

Figure 6. A: Irregularly shaped garnet (field of view $0.95 \mathrm{~mm}$ ). B: Hexagonalshaped sillimanite (field of view $1.3 \mathrm{~mm}$ ). C: Ellipsoidal diopside (field of view 1.6 $\mathrm{mm}$ ). D: Sapphirine (field of view $1.2 \mathrm{~mm}$ ). E: Rounded
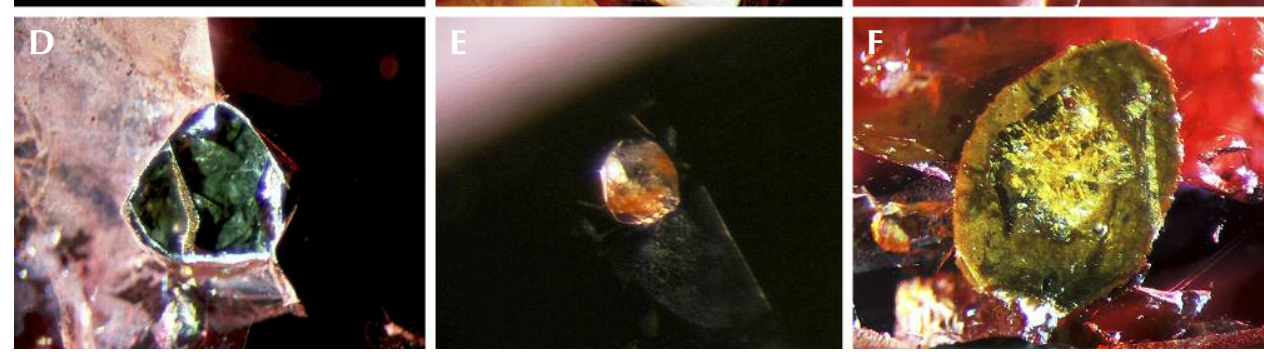
nepheline (field of view 0.8 mm). F: Quartz with an iron-stained edge (field of view $1.4 \mathrm{~mm}$ ). Photomicrographs by S. Promwongnan; brightfield illumination with fiber-optic light ( $A$ and $B)$ and darkfield illumination $(C-F)$.

nan and Sutthirat, 2019) and Pailin rubies (Sutherland et al., 1998b). However, diopsides found in Bo Welu ruby and purple sapphire had a wider range of composition, encompassing the compositions of diopside inclusions in both Bo Rai and Pailin rubies.

Sapphirine was recognized in a purple-red ruby (sample 9TWL160); it was a subhedral crystal surrounded by fractures (figure 6D) and showed strong green-blue pleochroism similar to that reported by Koivula and Fryer (1987). Its chemical composition (table 2) was similar to that of sapphirine inclusions found in alluvial rubies from the $\mathrm{Na}$ Wong gem field (Sutthirat et al., 2001; Saminpanya and Sutherland, 2011), located about $20 \mathrm{~km}$ south of Bo Welu (again, see figure 1). Sapphirine inclusions were also reported in ruby from Bo Rai (Koivula and Fryer, 1987) and Pailin (Sutherland et al., 1998b). Sapphirine inclusions in rubies from Bo $\mathrm{Na}$ Wong, Pailin, and $\mathrm{Bo}$ Welu all have similar mineral chemistry.
Nepheline inclusions were occasionally recognized in purple-red and reddish purple samples. They formed as rounded crystals (figure $6 \mathrm{E}$ ) and contained major contents of $\mathrm{SiO}_{2}$ and $\mathrm{Al}_{2} \mathrm{O}_{3}$ with traces of $\mathrm{CaO}$, $\mathrm{K}_{2} \mathrm{O}, \mathrm{Na}_{2} \mathrm{O}$, and $\mathrm{FeO}$ (table 2). This is the first report of nepheline inclusions in Thai ruby.

Quartz appeared to have formed as an exogenous inclusion in a purple-red ruby (sample 9TWL095). Its euhedral core was surrounded by an iron-stained yellow rim (figure $6 \mathrm{~F}$ ), and its composition was almost pure silica $\left(99.6 \% \mathrm{SiO}_{2}\right)$ with iron traces (table 2). The unusual quartz inclusion in Thai ruby is first reported here.

Feldspar inclusions were often observed in purple-red and reddish purple samples from Bo Welu. They usually formed as single grains with a rounded or irregular shape (figure 7A). Most feldspar inclusions were characterized by plagioclase compositions ranging widely from bytownite to andesine

Figure 7. Feldspar and spinel inclusions in Bo Welu ruby. A: Irregularly shaped feldspar (field of view $0.6 \mathrm{~mm}$, brightfield illumination). B: Feldspar-spinel composite (field of view $0.7 \mathrm{~mm}$, darkfield illumination). C: BSE image of the same inclusion in figure B revealing feldspar intergrowth with spinel (field of view $0.25 \mathrm{~mm}$ ). Photomicrographs by S. Promwongnan.
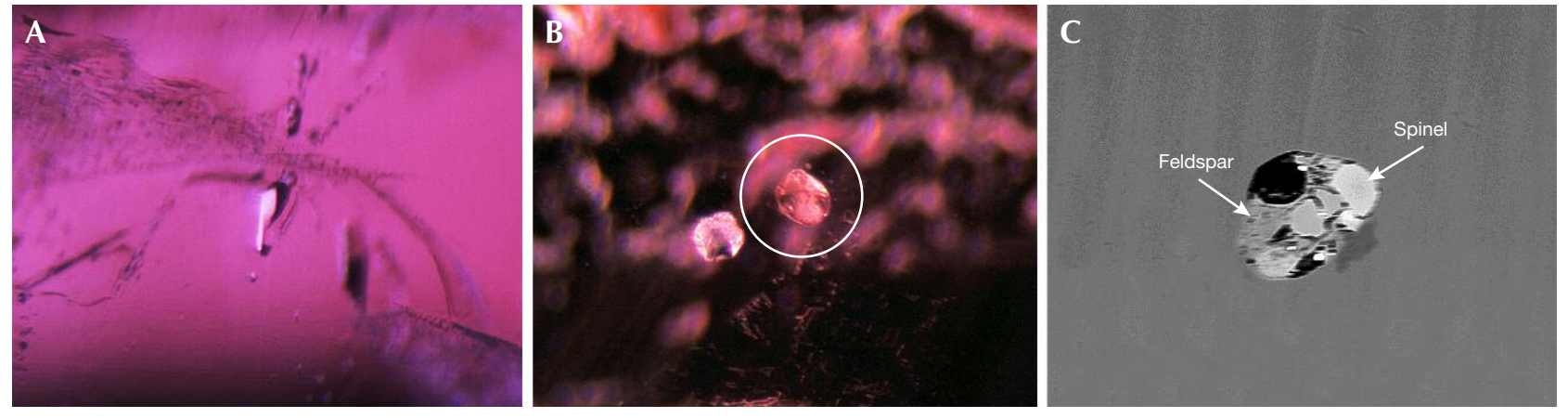
TABLE 3. EPMA analyses of feldspar inclusions in Bo Welu ruby and purple sapphire compared to blue sapphire samples.

\begin{tabular}{|c|c|c|c|c|c|c|c|c|c|}
\hline \multirow{4}{*}{$\begin{array}{l}\text { Mineral } \\
\text { phase } \\
\text { analysis } \\
\text { (wt.\%) }\end{array}$} & \multicolumn{5}{|c|}{ Ruby and Purple Sapphire } & \multirow{2}{*}{\multicolumn{4}{|c|}{$\begin{array}{l}\text { Blue Sapphire } \\
\text { Alkali feldspars }\end{array}$}} \\
\hline & \multicolumn{4}{|c|}{ Plagioclase feldspars } & \multirow{3}{*}{$\begin{array}{c}\text { Alkali } \\
\text { feldspars } \\
\text { rP } \\
\text { 9TWL012-2 }\end{array}$} & & & & \\
\hline & $\mathrm{PR}$ & rP & $\mathrm{rP}$ & PR & & \multirow{2}{*}{$\begin{array}{c}\text { gB } \\
\text { 8TWL083-2 }\end{array}$} & \multirow{2}{*}{$\begin{array}{c}\text { gB } \\
\text { 8TWL018-3 }\end{array}$} & \multirow{2}{*}{$\begin{array}{c}\text { B } \\
\text { 8TWL114-25 }\end{array}$} & \multirow{2}{*}{$\begin{array}{c}\text { gB } \\
\text { 8TWL093-5 }\end{array}$} \\
\hline & 9TWL055-1 & 9TWL122-4 & 9TWL176-1 & 9TWL154-1 ${ }^{\text {a }}$ & & & & & \\
\hline $\mathrm{SiO}_{2}$ & 58.02 & 48.61 & 61.34 & 55.99 & 64.91 & 66.19 & 66.97 & 66.47 & 69.26 \\
\hline $\mathrm{TiO}_{2}$ & 0.04 & nd & 0.07 & 0.03 & 0.03 & nd & 0.02 & 0.01 & 0.06 \\
\hline $\mathrm{Al}_{2} \mathrm{O}_{3}$ & 26.36 & 32.46 & 23.67 & 26.97 & 21.54 & 20.85 & 21.40 & 20.92 & 16.47 \\
\hline $\mathrm{FeO}$ & 0.09 & 0.07 & 0.46 & 0.25 & 0.12 & 0.08 & 0.65 & 0.59 & 0.31 \\
\hline $\mathrm{MnO}$ & 0.04 & 0.03 & nd & nd & 0.01 & nd & 0.04 & 0.04 & nd \\
\hline $\mathrm{MgO}$ & 0.05 & 0.03 & 0.20 & 0.03 & nd & nd & nd & 0.02 & nd \\
\hline $\mathrm{CaO}$ & 7.30 & 16.51 & 6.29 & 11.04 & 1.48 & 2.50 & 0.73 & 0.02 & 0.14 \\
\hline $\mathrm{Na}_{2} \mathrm{O}$ & 6.66 & 2.14 & 7.76 & 5.51 & 7.71 & 8.54 & 8.47 & 9.45 & 6.25 \\
\hline Total & 99.91 & 99.97 & 99.86 & 99.84 & 98.88 & 99.23 & 100.97 & 98.92 & 99.92 \\
\hline \multicolumn{10}{|c|}{ Formula $8(\mathrm{O})$} \\
\hline $\mathrm{Si}$ & 2.606 & 2.228 & 2.732 & 2.527 & 2.899 & 2.926 & 2.925 & 2.944 & 3.095 \\
\hline $\mathrm{Ti}$ & 0.001 & 0.000 & 0.002 & 0.001 & 0.001 & 0.000 & 0.001 & 0.000 & 0.002 \\
\hline $\mathrm{Al}$ & 1.396 & 1.753 & 1.243 & 1.435 & 1.134 & 1.086 & 1.102 & 1.092 & 0.868 \\
\hline $\mathrm{Fe}$ & 0.004 & 0.003 & 0.017 & 0.010 & 0.004 & 0.118 & 0.034 & 0.001 & 0.011 \\
\hline $\mathrm{Mn}$ & 0.002 & 0.001 & 0.000 & 0.000 & 0.000 & 0.003 & 0.024 & 0.022 & 0.000 \\
\hline$M g$ & 0.003 & 0.002 & 0.013 & 0.002 & 0.000 & 0.000 & 0.001 & 0.001 & 0.000 \\
\hline $\mathrm{Ca}$ & 0.352 & 0.811 & 0.300 & 0.534 & 0.071 & 0.000 & 0.000 & 0.001 & 0.007 \\
\hline K & 0.077 & 0.007 & 0.004 & 0.001 & 0.175 & 0.060 & 0.150 & 0.079 & 0.424 \\
\hline $\mathrm{Na}$ & 0.580 & 0.190 & 0.670 & 0.482 & 0.668 & 0.732 & 0.718 & 0.811 & 0.542 \\
\hline \multicolumn{10}{|c|}{ Atomic\% } \\
\hline $\mathrm{Ca}$ & 34.86 & 80.47 & 30.82 & 52.46 & 7.74 & 13.01 & 3.79 & 0.10 & 0.69 \\
\hline $\mathrm{K}$ & 7.66 & 0.67 & 0.41 & 0.12 & 19.18 & 6.62 & 16.62 & 8.89 & 43.58 \\
\hline $\mathrm{Na}$ & 57.49 & 18.85 & 68.77 & 47.42 & 73.08 & 80.37 & 79.60 & 91.01 & 55.72 \\
\hline Total & 100.00 & 100.00 & 100.00 & 100.00 & 100.00 & 100.00 & 100.00 & 100.00 & 100.00 \\
\hline \multicolumn{10}{|c|}{$\begin{array}{l}{ }^{a} \text { Feldspar combined } \text { with spinel inclusion } \\
{ }^{b} \text { Feldspar combined with monazite inclusion } \\
\text { nd }=\text { not detected } \\
P R=\text { purple-red, } r P=\text { reddish purple, } P=\text { purple, } g B=\text { greenish blue, } B=\text { blue }\end{array}$} \\
\hline
\end{tabular}

$\left(\mathrm{Ab}_{19-69} \mathrm{An}_{31-80} \mathrm{Or}_{<8}\right.$ see table 3 and figure 8). Moreover, an unusual rounded alkali feldspar of anorthoclase composition $\left(\mathrm{Ab}_{73} \mathrm{An}_{8} \mathrm{Or}_{19}\right)$ was recognized in reddish purple sample 9TWL012; its Raman spectrum was similar to other feldspar inclusions, but the peaks were shifted slightly to the right. Feldspar-spinel composite inclusions were also observed in ruby and purple sapphire (samples 9TWL019, 9TWL154, and 9TWL180); their compositions fell mostly within the labradorite range $\left(\mathrm{Ab}_{38-48} \mathrm{An}_{51-62} \mathrm{Or}_{0.01-3}\right)$. The composite feldspar and colorless spinel inclusions were found in a few ruby samples such as purple-red sample 9TWL154 (figure 7B), which can be observed clearly under backscattered-electron (BSE) images (figure 7C). The composition range of feldspar inclusions in Bo Welu ruby and purple sapphire was wider than that found in Bo Rai ruby, which indicates only bytownite $\left(\mathrm{Ab}_{11-15} \mathrm{An}_{85-89} \mathrm{Or}_{0.1-0.2}\right)$ and andesine $\left(\mathrm{Ab}_{56} \mathrm{An}_{38} \mathrm{Or}_{6}\right)$ (Promwongnan and Sutthirat, 2019). Although Gübelin (1971) and Promwongnan and Sutthirat (2019) previously reported plagioclase feldspar inclusions in Thai ruby and purple sapphire, this is the first report of them containing a feldspar-spinel composite inclusion as well as alkali feldspar.

Apart from spinel-feldspar composite inclusions, a single tiny rounded spinel inclusion was observed in a 


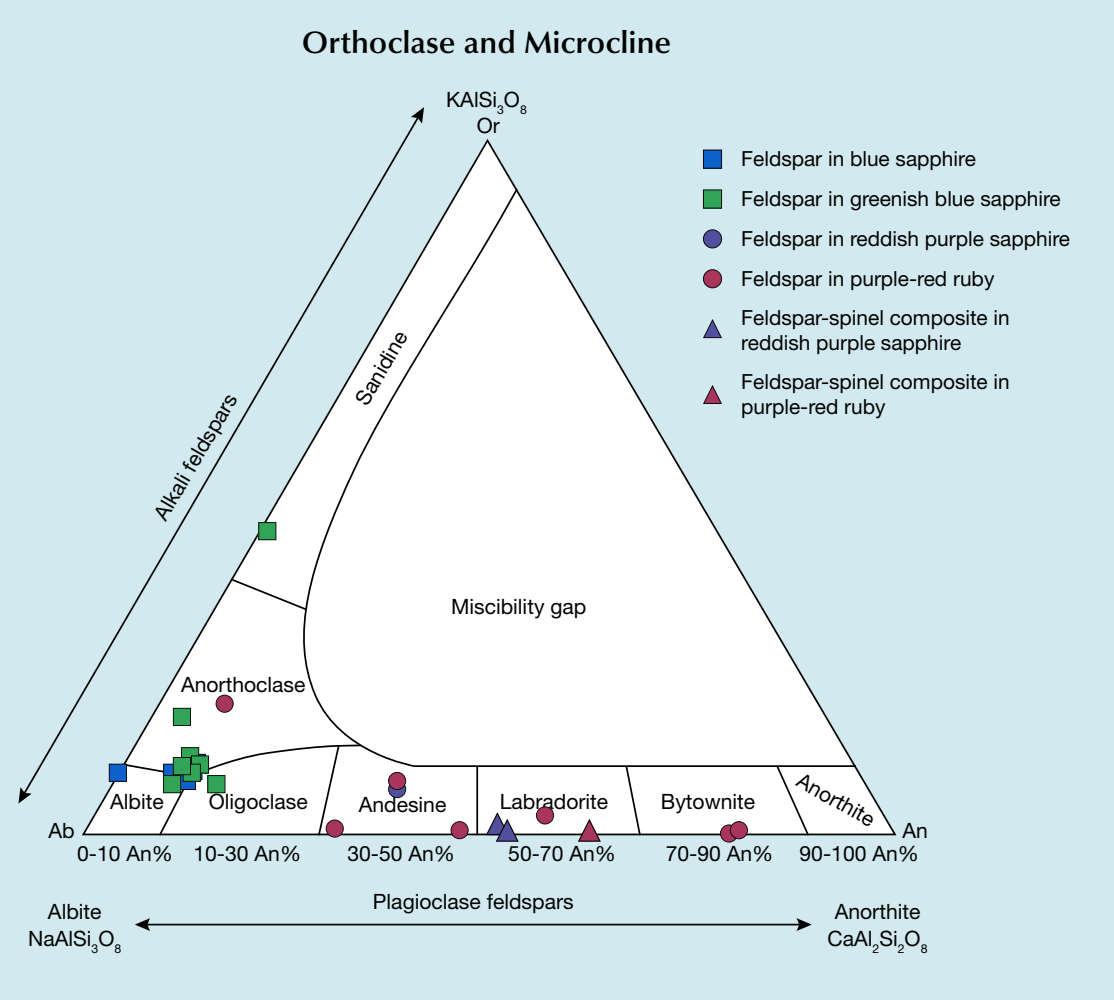

Figure 8. Ternary plot of feldspar inclusions in rubies and sapphires from Bo Welu. Most feldspar inclusions in the ruby and purple sapphire samples fell within the range of plagioclase. One with anorthoclase composition was recognized in a reddish purple sapphire (9TWL012). Most feldspar inclusions in the blue sapphire samples were alkali feldspar. Only one sanidine inclusion was recorded, in a greenish blue sapphire (8TWL093).

purple-red sample (9TWL085). All spinel inclusions appeared to have similar composition, with high contents of $\mathrm{Al}$ and $\mathrm{Mg}$ greater than $\mathrm{Fe}$ (average $\mathrm{Mg}_{0.77} \mathrm{Fe}_{0.23} \mathrm{Al}_{2} \mathrm{O}_{4}$ or $\mathrm{Spl}_{77} \mathrm{Hc}_{23}$; table 4). Recently, a spinel inclusion with a composition of $\mathrm{Mg}_{0.83} \mathrm{Fe}_{0.17} \mathrm{Al}_{2} \mathrm{O}_{4}$ (or $\mathrm{Spl}_{83} \mathrm{Hc}_{17}$ ) was reported in ruby and purple sapphire from Bo Rai (Promwongnan and Sutthirat, 2019). Moreover, a pleonaste spinel inclusion was reported in ruby from West Pailin, Cambodia (Sutherland et al., 1998b), with Mg content clearly lower than the spinel inclusions in Thai ruby.
Sulfide was sometimes observed as subhexagonal or rounded metallic opaque inclusions (figure 9A). Their compositions clearly did not match with the ideal formula of pyrrhotite (FeS), but they also contained additional nickel and copper, similar to sulfide inclusions in Bo Rai ruby and purple sapphire reported by Promwongnan and Sutthirat (2019). Moreover, subhexagonal to rounded opaque metallic chalcopyrite $\left(\mathrm{CuFeS}_{2}\right)$ inclusions in Thai rubies were reported by Gübelin (1971).

Figure 9. A: Rounded sulfide inclusion surrounded by a tension disk (field of view $0.95 \mathrm{~mm}$ ). B: Euhedral anhydrite containing subhedral black sulfide (pyrrhotite) (field of view $0.8 \mathrm{~mm}$ ). C: Rounded two-phase inclusion containing a gas bubble and silica melt (field of view $1.0 \mathrm{~mm}$ ). Photomicrographs by S. Promwongnan; darkfield illumination with fiber-optic light (A) and brightfield illumination with fiber-optic light (B-C).
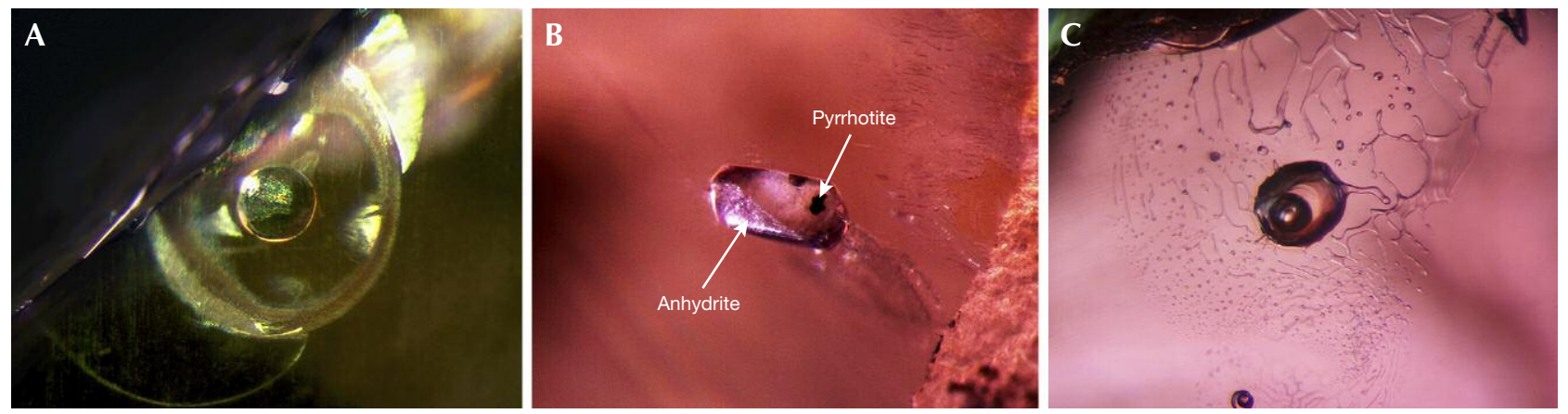
TABLE 4. EPMA analyses of spinel, anhydrite, and sulfide inclusions in Bo Welu ruby and purple sapphire samples.

\begin{tabular}{|c|c|c|c|c|c|c|}
\hline \multirow{2}{*}{$\begin{array}{l}\text { Mineral } \\
\text { phase } \\
\text { analysis } \\
\text { (wt.\%) }\end{array}$} & \multicolumn{4}{|c|}{ Spinel } & Anhydrite & Sulfide \\
\hline & $\begin{array}{c}\text { PR } \\
\text { 9TWL019-2a }\end{array}$ & $\begin{array}{c}\text { PR } \\
\text { 9TWL154-2 }\end{array}$ & $\begin{array}{c}\text { rP } \\
\text { 9TWL180-2a }\end{array}$ & $\begin{array}{c}\text { PR } \\
\text { 9TWL085-2 }\end{array}$ & \multicolumn{2}{|c|}{$\begin{array}{c}\text { rP } \\
\text { 9TWL096 }\end{array}$} \\
\hline $\mathrm{SO}_{3}$ & na & na & na & na & 55.62 & 53.72 \\
\hline $\mathrm{SiO}_{2}$ & 0.04 & 0.10 & 0.06 & 0.09 & 0.03 & 0.02 \\
\hline $\mathrm{TiO}_{2}$ & 0.43 & 2.51 & 1.09 & nd & nd & 0.01 \\
\hline $\mathrm{Al}_{2} \mathrm{O}_{3}$ & 64.71 & 63.37 & 64.25 & 67.46 & 0.03 & 0.03 \\
\hline $\mathrm{Cr}_{2} \mathrm{O}_{3}$ & 0.25 & 1.67 & 0.13 & 0.38 & nd & 0.09 \\
\hline FeO total & 13.11 & 10.61 & 13.50 & 11.81 & 0.02 & 44.97 \\
\hline $\mathrm{MnO}$ & 0.09 & 0.24 & 0.10 & 0.27 & 0.06 & nd \\
\hline $\mathrm{MgO}$ & 20.19 & 20.59 & 20.56 & 19.96 & 0.01 & 0.01 \\
\hline $\mathrm{ZnO}$ & 0.09 & 0.04 & 0.04 & na & na & na \\
\hline $\mathrm{CaO}$ & nd & 0.01 & 0.04 & 0.03 & 44.23 & 1.12 \\
\hline $\mathrm{K}_{2} \mathrm{O}$ & nd & nd & 0.02 & nd & nd & 0.02 \\
\hline $\mathrm{Na}_{2} \mathrm{O}$ & nd & nd & 0.03 & nd & 0.02 & nd \\
\hline Total & 98.91 & 99.14 & 99.82 & 100.00 & 100.00 & 99.99 \\
\hline Formula & & & & & $4(\mathrm{O})$ & - \\
\hline S & - & - & - & - & 0.966 & 1.008 \\
\hline $\mathrm{Si}$ & 0.001 & 0.002 & 0.002 & 0.002 & 0.001 & 0.000 \\
\hline $\mathrm{Ti}$ & 0.008 & 0.048 & 0.021 & 0.000 & 0.000 & 0.000 \\
\hline $\mathrm{Al}$ & 1.948 & 1.893 & 1.921 & 1.990 & 0.001 & 0.001 \\
\hline $\mathrm{Cr}$ & 0.005 & 0.033 & 0.003 & 0.007 & 0.000 & 0.002 \\
\hline${ }^{\mathrm{b}} \mathrm{Fe}^{3+}$ & 0.038 & 0.000 & 0.044 & 0.000 & 0.000 & 0.940 \\
\hline${ }^{\mathrm{b}} \mathrm{Fe}^{2+}$ & 0.242 & 0.225 & 0.242 & 0.247 & 0.000 & \\
\hline $\mathrm{Mn}$ & 0.002 & 0.005 & 0.002 & 0.006 & 0.001 & 0.000 \\
\hline$M g$ & 0.769 & 0.778 & 0.778 & 0.745 & 0.000 & 0.001 \\
\hline $\mathrm{Zn}$ & 0.002 & 0.001 & 0.001 & - & - & - \\
\hline $\mathrm{Ca}$ & 0.000 & 0.000 & 0.001 & 0.001 & 1.097 & 0.030 \\
\hline K & 0.000 & 0.000 & 0.001 & 0.000 & 0.000 & 0.001 \\
\hline $\mathrm{Na}$ & 0.000 & 0.000 & 0.001 & 0.000 & 0.001 & 0.000 \\
\hline Total & 3.014 & 2.986 & 3.017 & 2.999 & 2.067 & 1.982 \\
\hline
\end{tabular}

a Spinel composition with feldspar inclusion

${ }^{b} \mathrm{Fe}^{2+}$ and $\mathrm{Fe}^{3+}$ recalculated from total FeO after the method of Droop (1987) and assigned using ideal formula cations nd $=$ not detected

na $=$ not analyzed

$P R=$ purple-red, $r P=$ reddish purple

An anhydrite-sulfide composite inclusion was found in a reddish purple sapphire (9TWL096). The anhydrite formed a subhedral crystal containing an irregularly shaped sulfide (figure 9B). This sulfide is chemically characterized by a pure pyrrhotite composition $\left(\mathrm{Fe}_{0.94} \mathrm{~S}\right.$; see table 4). This is the first report of an anhydrite inclusion in Thai reddish purple sapphire.

Two-phase inclusions were sometimes observed in Bo Welu ruby and purple sapphire samples. Rounded two-phase inclusions, consisting of a gas bubble and silicate melt, were situated within healed fractures (figure 9C). The silicate melts contained major contents of $\mathrm{SiO}_{2}$ and $\mathrm{Al}_{2} \mathrm{O}_{3}$ with traces of $\mathrm{CaO}$, $\mathrm{FeO}$, and $\mathrm{MgO}$.

Mineral Inclusions in Blue Sapphire. The most common internal features in Bo Welu blue sapphire were strong color zoning (figure 10A) and orientation of fine minute particles (figure 10B). Other features such as oriented silk inclusions, thin films (figure 

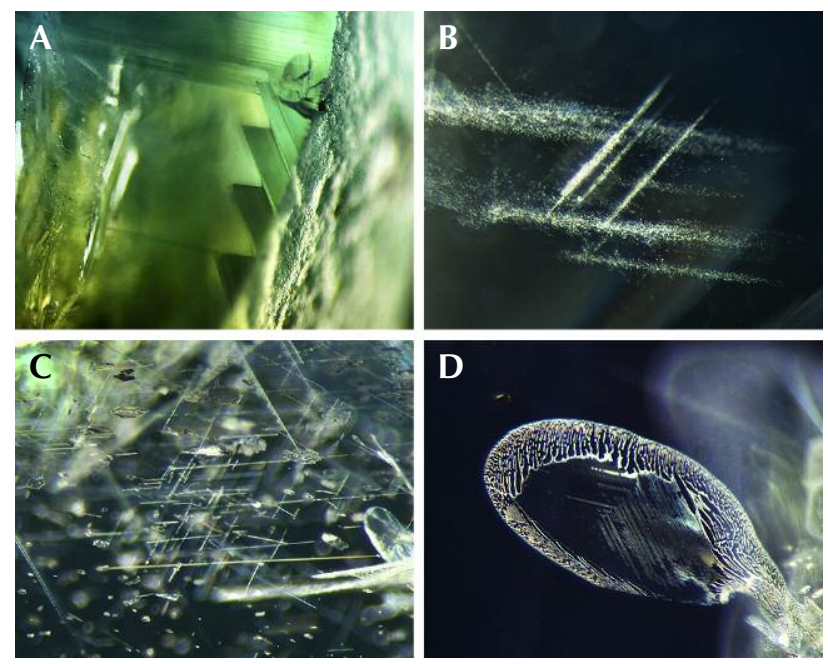

Figure 10. Common internal features found in Bo Welu blue sapphire, viewed using a combination of fiber-optic with darkfield illumination. A: Strong color zoning (field of view $2.3 \mathrm{~mm}$ ). B: Oriented fine minute particles (field of view $1.3 \mathrm{~mm}$ ). C: Oriented silk inclusions and a thin film (field of view $1.6 \mathrm{~mm}$ ). D: Healed fractures (field of view $1.2 \mathrm{~mm}$ ). Photomicrographs by S. Promwongnan.

10C), and healed fractures (figure 10D) could be observed clearly under darkfield illumination. Moreover, varieties of mineral inclusions were also recognized in these samples. Some had never been documented in blue sapphire from either Thailand or Pailin, Cambodia (see table 1).

Zircon characterized by tetragonal prismatic (figure 11A) or bipyramidal crystals (figure 11B), in this case displaying stress fractures, was sometimes observed in the blue sapphires. They had a narrow compositional range of about $2-3 \mathrm{wt}$. $\% \mathrm{HfO}_{2}, \leq 1.1 \mathrm{wt}$. $\%$

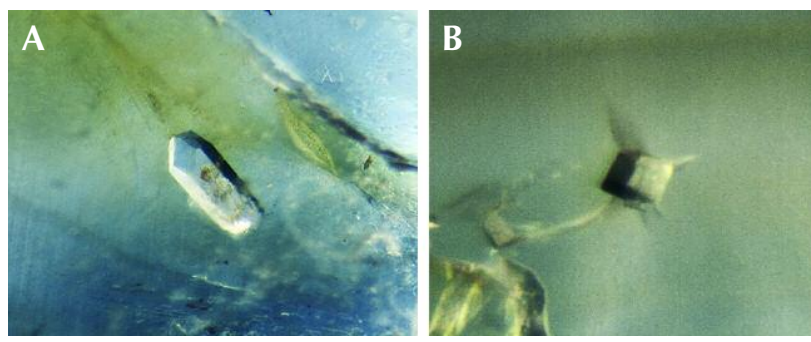

Figure 11. A: Prismatic zircon inclusion in blue sapphire (field of view $1.0 \mathrm{~mm}$ ). B: Bipyramidal zircon without pyramidal face associated with stress fissure (field of view $0.2 \mathrm{~mm}$ ). Photomicrographs by S. Promwongnan; darkfield illumination.

$\mathrm{UO}_{2}$, mostly $<1.2$ wt. $\% \mathrm{ThO}_{2},<0.9$ wt. $\% \mathrm{Y}_{2} \mathrm{O}_{3^{\prime}}$ and $<1.1 \mathrm{wt} . \%$ rare earth elements (see table 5 ). The zircon inclusions in this study were similar to those in sapphire from Bo Phloi, Kanchanaburi (Saminpanya and Sutherland, 2011; Khamloet et al., 2014), but they contained higher $\mathrm{HfO}_{2}$ content than those from Kao Wua, Chanthaburi (Sutherland et al., 1998a).

Feldspar was the most common inclusion in Bo Welu blue sapphire, typically formed as euhedral to subhedral crystals (figure 12A). Some were also associated with healed fractures or tension cracks (figure 12B). Most of the feldspar inclusions contained a Narich (albite) component with low $\mathrm{K}$ and $\mathrm{Ca}$ contents ranging between $\mathrm{Ab}_{80-91} \mathrm{An}_{0.1-13} \mathrm{Or}_{7-17}$ (table 3, figure 8); surprisingly, feldspar composition $\left(\mathrm{Ab}_{56} \mathrm{An}_{0.7} \mathrm{Or}_{44}\right)$ was determined from a subhedral inclusion surrounded by a tension crack in greenish blue sapphire sample 8TWL093 (figure 12C) that displayed a Raman spectrum matching that of sanidine. Albite and sanidine inclusions were previously reported in Bo Phloi sapphire in Kanchanaburi and Den Chai

Figure 12. A: Euhedral feldspar inclusion in blue sapphire (field of view $0.8 \mathrm{~mm}$, darkfield illumination). B: Feldspar crystals situated in healed fractures (field of view $0.6 \mathrm{~mm}$, darkfield illumination). C: Subhedral sanidine inclusion surrounded by a tension crack (field of view $0.7 \mathrm{~mm}$, brightfield illumination). Photomicrographs by S. Promwongnan.
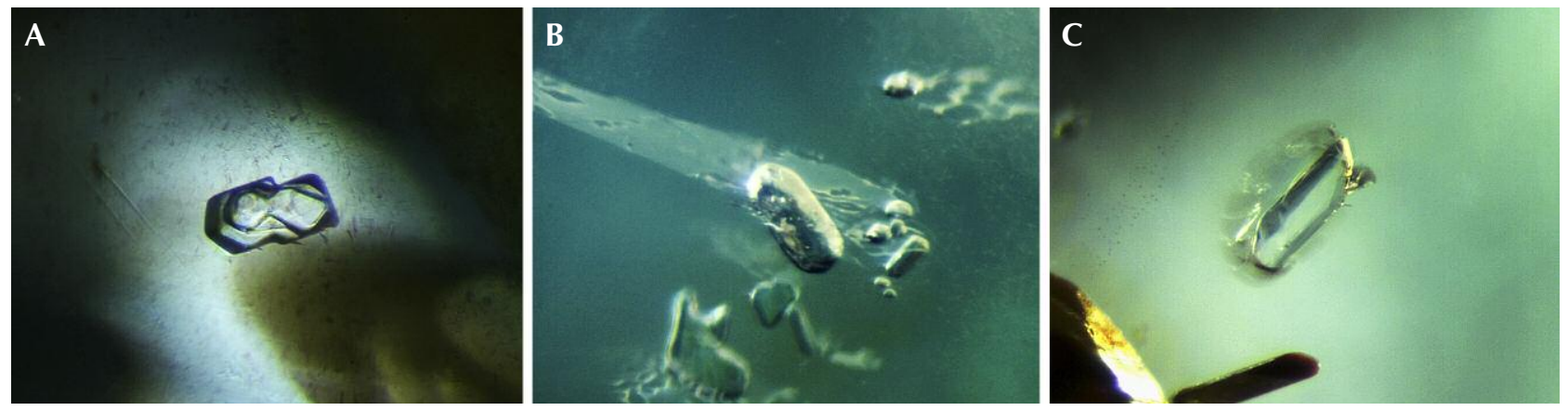

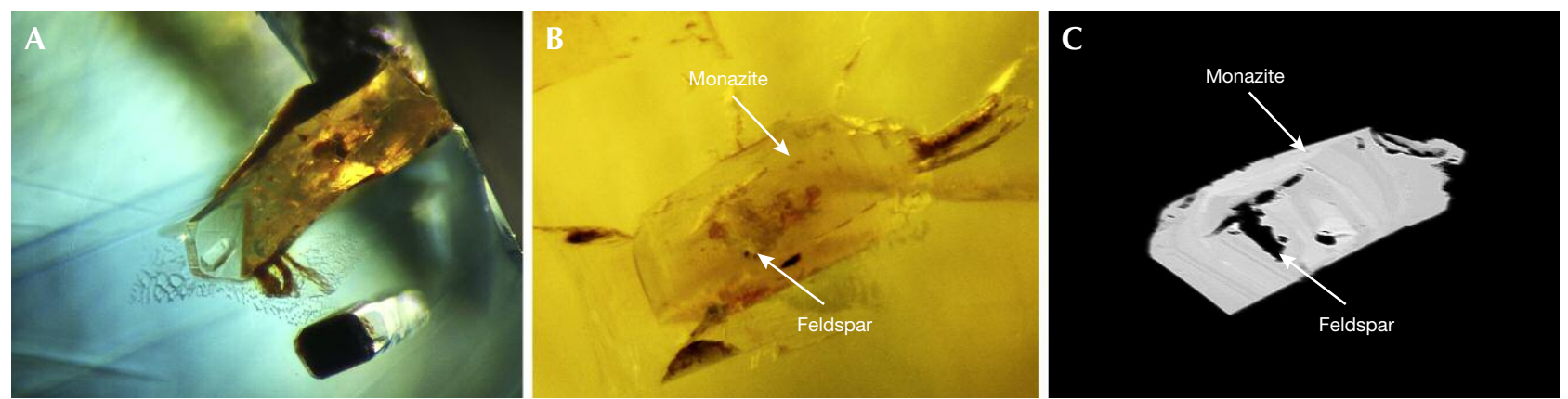

Figure 13. A: Colorless subhedral monazite crystal (field of view $1.0 \mathrm{~mm}$ ). B: Monazite inclusion containing albite feldspar (field of view $0.7 \mathrm{~mm}$ ). C: BSE image of the same inclusion in figure B (field of view $0.65 \mathrm{~mm}$ ). Photomicrographs by S. Promwongnan, brightfield illumination.

sapphire in Phrae Province (Saminpanya and Sutherland, 2011; Khamloet et al., 2014).

Monazite was observed in some of the blue sapphire samples. These were typically colorless to very light yellow crystals that usually occurred as prisms (figure 13A). An unusual composite inclusion combining monazite and albite feldspar $\left(\mathrm{Ab}_{8.9} \mathrm{An}_{0.1} \mathrm{Or}_{91.0}\right.$ see table 3) was recognized in sample 8TWL114 (figure $13 \mathrm{~B})$, clearly visible with BSE imaging (figure 13C). Most of the monazite inclusions varied in composition from about 35-46 wt. \% $\mathrm{ThO}_{2}, 15-24 \mathrm{wt} . \% \mathrm{Ce}_{2} \mathrm{O}_{3}$, and 16-22 wt. \% $\mathrm{P}_{2} \mathrm{O}_{5}$ (see table 5). Monazite inclusions in sapphire have also been reported in basaltic sapphires from southwest Rwanda (Krzemnicki et al., 1996), Tok Phrom in Chanthaburi and Bo Phloi in Kanchanaburi (Intasopa et al., 1998; Khamloet et al., 2014), and Laos (Singbamroong and Thanasuthipitak, 2004).

Columbite was often found in the blue sapphire samples, usually as black grains with an irregular or flat shape or as orthorhombic crystals (figure 14A), with some surrounded by tension cracks. Some dark red columbite inclusions also presented comet-like dust trails (figure 14B). Their compositions varied in narrow ranges of $74-75 \mathrm{wt} . \% \mathrm{Nb}_{2} \mathrm{O}_{5^{\prime}} \leq 0.3 \mathrm{wt}$. $\% \mathrm{Ta}_{2} \mathrm{O}_{5}$ $\leq 0.4$ wt. $\% \mathrm{TiO}_{2}, \leq 0.2 \mathrm{wt} . \% \mathrm{MgO}$, and $<10.5$ wt. $\% \mathrm{FeO}$ (table 5). Their compositions are plotted on the $\mathrm{FeTa}_{2} \mathrm{O}_{6}-\mathrm{FeNb}_{2} \mathrm{O}_{6}-\mathrm{MnNb}_{2} \mathrm{O}_{6}-\mathrm{MnTa}_{2} \mathrm{O}_{6}$ quadrilateral diagram for columbite-tantalite classification (Černý and Ercit, 1985; see figure 15). The recalculated atomic formula of these columbite inclusions, based on six oxygens, seems to have some cations missing; they fall distinctly in the field of ferro-columbite. Their compositions have less $\mathrm{Ti}, \mathrm{Ta}$, and $\mathrm{Fe}$ oxides than columbite inclusions in basaltic sapphires from New South Wales in Australia, previously reported by Sutherland et al. (1998a). Moreover, columbite inclusions in sapphire from Tok Phrom in Chanthaburi and Pailin in Cambodia were also reported by Intasopa et al. (1998).

Sulfide was rarely found as an inclusion in the blue sapphire samples; however, some small black

Figure 14. A: Subhedral columbite inclusion (field of view $1.0 \mathrm{~mm}$, brightfield illumination). B: Dark red columbite inclusions presenting comet-like dust trails (field of view $0.8 \mathrm{~mm}$, darkfield illumination). C: Tiny black opaque sulfide inclusions, shown using fiber-optic reflection (field of view $1.0 \mathrm{~mm}$ ). Photomicrographs by S. Promwongnan.
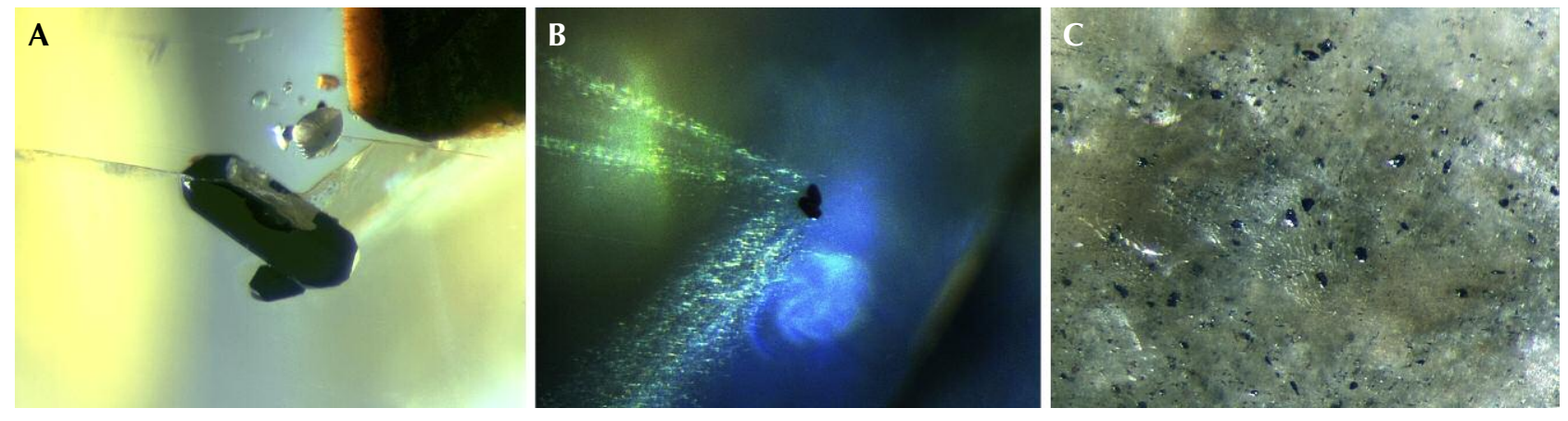
TABLE 5. EPMA analyses of zircon, monazite, and columbite inclusions in Bo Welu blue sapphire samples.

\begin{tabular}{|c|c|c|c|c|c|c|c|c|c|c|}
\hline \multirow{2}{*}{$\begin{array}{l}\text { Mineral } \\
\text { phase } \\
\text { analysis } \\
\text { (wt.\%) }\end{array}$} & \multicolumn{3}{|c|}{ Zircon } & \multicolumn{4}{|c|}{ Monazite } & \multicolumn{3}{|c|}{ Columbite } \\
\hline & $\begin{array}{c}\text { B } \\
\text { 8TWL040-2 }\end{array}$ & $\begin{array}{c}\text { B } \\
\text { 8TWL059-2 }\end{array}$ & $\begin{array}{c}\mathrm{gB} \\
\text { 8TWL059-6 }\end{array}$ & $\begin{array}{c}\text { gB } \\
\text { 8TWL036-4 }\end{array}$ & $\begin{array}{c}\text { gB } \\
\text { 8TWL036-7 }\end{array}$ & $\begin{array}{c}\text { gB } \\
\text { 8TWL082-7 }\end{array}$ & $\begin{array}{c}\text { B } \\
8 T W L 114-24^{a} \\
\end{array}$ & $\begin{array}{c}\text { gB } \\
\text { 8TWL064-1 }\end{array}$ & $\begin{array}{c}\text { gB } \\
\text { 8TWL036-2 }\end{array}$ & $\begin{array}{c}\mathrm{gB} \\
\text { 8TWL097-7 }\end{array}$ \\
\hline $\mathrm{Nb}_{2} \mathrm{O}_{5}$ & na & na & na & na & 0.12 & 0.01 & nd & 74.37 & 74.39 & 74.57 \\
\hline $\mathrm{P}_{2} \mathrm{O}_{5}$ & nd & 0.57 & 0.49 & 15.81 & 21.62 & 18.72 & 18.14 & na & na & na \\
\hline $\mathrm{Ta}_{2} \mathrm{O}_{5}$ & na & na & na & na & 0.51 & 0.15 & 0.41 & 0.25 & 0.31 & 0.16 \\
\hline $\mathrm{HfO}_{2}$ & 2.09 & 2.62 & 2.80 & na & 0.13 & nd & 0.28 & na & na & na \\
\hline $\mathrm{SiO}_{2}$ & 34.78 & 33.63 & 33.86 & 3.71 & 4.59 & 5.31 & 4.80 & na & na & na \\
\hline $\mathrm{ThO}_{2}$ & 0.07 & 1.21 & 0.47 & 46.46 & 35.15 & 45.12 & 38.14 & 0.85 & 0.87 & 0.11 \\
\hline $\mathrm{TiO}_{2}$ & na & na & na & na & na & na & na & 0.43 & 0.37 & 0.40 \\
\hline $\mathrm{UO}_{2}$ & 0.24 & 1.13 & 0.75 & 0.95 & nd & nd & 0.05 & 3.50 & 3.45 & 2.10 \\
\hline $\mathrm{ZrO}_{2}$ & 61.13 & 59.39 & 60.66 & na & 0.02 & nd & nd & 0.78 & 0.57 & 0.83 \\
\hline $\mathrm{Al}_{2} \mathrm{O}_{3}$ & 0.07 & 0.01 & nd & 0.07 & nd & nd & nd & 0.29 & 0.15 & 0.14 \\
\hline $\mathrm{Ce}_{2} \mathrm{O}_{3}$ & na & na & na & 19.28 & 24.32 & 15.38 & 24.30 & 1.36 & 1.27 & 1.85 \\
\hline $\mathrm{Gd}_{2} \mathrm{O}_{3}$ & na & na & na & 1.14 & 0.57 & 0.92 & 0.71 & na & na & na \\
\hline $\mathrm{La}_{2} \mathrm{O}_{3}$ & na & na & na & 1.04 & nd & nd & nd & na & na & na \\
\hline $\mathrm{Nd}_{2} \mathrm{O}_{3}$ & 0.02 & nd & nd & 5.33 & 6.94 & 7.33 & 6.41 & 1.42 & 1.46 & 1.93 \\
\hline $\mathrm{Pr}_{2} \mathrm{O}_{3}$ & nd & nd & 0.97 & 3.04 & 4.50 & 2.80 & 4.80 & na & na & na \\
\hline $\mathrm{Sm}_{2} \mathrm{O}_{3}$ & na & na & na & 0.80 & 0.24 & 0.20 & 0.12 & 0.72 & 0.76 & 0.77 \\
\hline $\mathrm{Y}_{2} \mathrm{O}_{3}$ & 0.17 & 0.85 & 0.81 & 0.08 & 0.22 & 0.99 & 0.48 & 2.76 & 3.19 & 3.46 \\
\hline $\mathrm{FeO}$ & 0.08 & 0.07 & 0.08 & 0.09 & 0.20 & 0.07 & nd & 9.79 & 10.37 & 10.45 \\
\hline $\mathrm{MnO}$ & na & na & na & na & na & na & na & 1.58 & 1.48 & 1.24 \\
\hline $\mathrm{MgO}$ & na & na & na & na & na & na & na & 0.08 & 0.09 & 0.16 \\
\hline $\mathrm{CaO}$ & na & na & na & 0.24 & 0.24 & 2.19 & 1.70 & 0.42 & 0.47 & 0.81 \\
\hline $\mathrm{Na}_{2} \mathrm{O}$ & na & na & na & na & na & na & na & nd & nd & nd \\
\hline Total & 98.71 & 99.57 & 101.01 & 98.64 & 99.40 & 99.39 & 100.41 & 98.61 & 99.18 & 98.97 \\
\hline Formula & & $4(O)$ & & & $4(O)$ & & & $6(O)$ & & \\
\hline $\mathrm{Ta}$ & - & - & - & - & 0.006 & 0.002 & 0.005 & 0.00 & 0.01 & 0.00 \\
\hline $\mathrm{Hf}$ & 0.018 & 0.023 & 0.024 & - & 0.002 & 0.000 & 0.004 & - & - & - \\
\hline $\mathrm{Si}$ & 1.062 & 1.035 & 1.027 & 0.186 & 0.199 & 0.240 & 0.219 & - & - & - \\
\hline Th & 0.000 & 0.008 & 0.003 & 0.529 & 0.347 & 0.463 & 0.395 & 0.011 & 0.012 & 0.001 \\
\hline $\mathrm{Ti}$ & - & - & - & - & - & - & 0.000 & 0.019 & 0.016 & 0.018 \\
\hline$U$ & 0.002 & 0.008 & 0.005 & 0.011 & 0.000 & 0.000 & 0.000 & 0.046 & 0.045 & 0.027 \\
\hline $\mathrm{Zr}$ & 0.910 & 0.891 & 0.897 & - & 0.000 & 0.000 & 0.000 & 0.022 & 0.016 & 0.023 \\
\hline $\mathrm{Al}$ & 0.003 & 0.000 & 0.000 & 0.004 & 0.000 & 0.000 & 0.000 & 0.020 & 0.010 & 0.010 \\
\hline $\mathrm{Ce}$ & - & - & - & 0.353 & 0.387 & 0.254 & 0.405 & 0.029 & 0.027 & 0.039 \\
\hline Dy & 0.001 & 0.001 & 0.001 & 0.010 & 0.001 & 0.003 & 0.001 & - & - & - \\
\hline Gd & - & - & - & 0.019 & 0.008 & 0.014 & 0.011 & - & - & - \\
\hline La & - & - & - & 0.019 & 0.000 & 0.000 & 0.000 & - & - & - \\
\hline $\mathrm{Nd}$ & 0.000 & 0.000 & 0.000 & 0.095 & 0.108 & 0.118 & 0.104 & 0.030 & 0.030 & 0.040 \\
\hline $\operatorname{Pr}$ & 0.000 & 0.000 & 0.011 & 0.055 & 0.071 & 0.046 & 0.080 & - & - & - \\
\hline $\mathrm{Sm}$ & - & - & - & 0.014 & 0.004 & 0.003 & 0.002 & 0.015 & 0.015 & 0.015 \\
\hline Y & 0.003 & 0.014 & 0.013 & 0.002 & 0.005 & 0.024 & 0.012 & 0.086 & 0.099 & 0.107 \\
\hline $\mathrm{Fe}$ & 0.002 & 0.002 & 0.002 & 0.004 & 0.007 & 0.003 & 0.000 & 0.481 & 0.507 & 0.508 \\
\hline $\mathrm{Mn}$ & - & - & - & - & - & - & - & 0.079 & 0.073 & 0.061 \\
\hline $\mathrm{Mg}$ & - & - & - & - & - & - & - & 0.007 & 0.008 & 0.014 \\
\hline $\mathrm{Ca}$ & - & - & - & 0.013 & 0.011 & 0.106 & 0.083 & 0.026 & 0.030 & 0.051 \\
\hline $\mathrm{Na}$ & - & - & - & - & - & - & - & 0.000 & 0.000 & 0.000 \\
\hline Total & 2.001 & 1.995 & 1.998 & 1.983 & 1.954 & 1.990 & 2.019 & 2.847 & 2.861 & 2.879 \\
\hline
\end{tabular}

${ }^{a}$ Monazite combined with feldspar inclusion

nd $=$ not detected: na $=$ not analyzed

$B=$ blue, $g B=$ greenish blue 


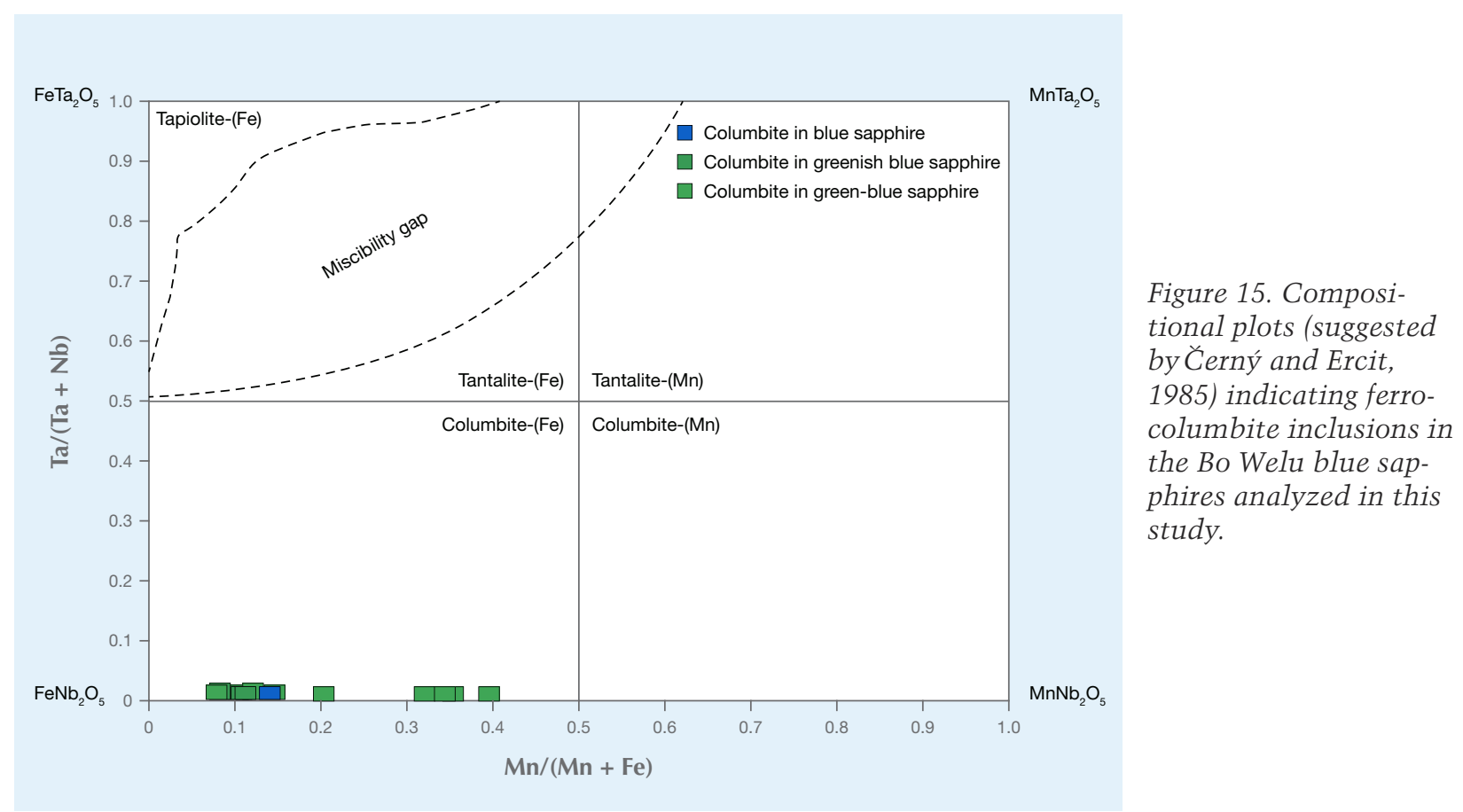

opaque crystals oriented along the crystallographic direction of the host sapphire, sample 8TWL046 (figure 14C), were recognized as sulfide. Their composition $\left(\mathrm{FeS}_{1.1}\right)$ was close to the ideal pyrrhotite formula and similar to sulfide in sapphire from New England in Australia (Sutherland et al., 1998a). On the other hand, these compositions are different from sulfide inclusions in sapphire from New South Wales, which contains trace compositions of $\mathrm{Cu}$ and $\mathrm{Zn}$ (Guo et al., 1996). However, it must be noted that this is the first discovery of sulfide inclusions in Thai blue sapphire.

\section{DISCUSSION}

Alluvial ruby and sapphire from the Bo Welu area showed characteristics of basaltic-type origin, particularly surface etching by carrier magmas.

Mineral inclusions in Thai corundum have been documented for almost eight decades (Gübelin, 1940). In this study, high-alumina diopside was the most common mineral inclusion in ruby and purple sapphire from Bo Welu. Mineral inclusions of pyrope garnet, sapphirine, quartz, feldspar, and sulfide were also identified. These inclusions have been mentioned in earlier studies on Thai ruby (e.g., Gübelin, 1940; Koivula and Fryer, 1987; Sutthirat et al., 2001; Saminpanya and Sutherland, 2011). Sillimanite, nepheline, quartz, alkali feldspar, spinel, and anhydrite are newly reported in this study as inclusions in ruby and purple sapphire from Bo Welu; this is also the first report for most of them in any such samples from Thailand. These minerals can provide more information about the environment of the host's crystallization before they traveled rapidly to the surface during magmatic eruption. Sillimanite, spinel, and melt inclusions, reported recently in Bo Rai ruby and purple sapphire (Promwongnan and Sutthirat, 2019), offer new evidence to support a hightemperature environment of the original host's formation in the following discussion.

Cr-rich content in the host and Al-rich content in mafic mineral inclusions such as pyroxene, pyrope garnet, plagioclase, nepheline, and sapphirine indicate a deep-seated source (most likely mantle or lower crust). Pyroxenes are inosilicate minerals and a key constituent of mafic igneous rocks. High-alumina diopside usually occurs in granulitic xenoliths in alkali basalts (Sutherland et al., 2003). Pyrope-rich endmember garnet inclusions are related to a liquid magmatic occurrence (Gübelin and Koivula, 1986). More than $55 \%$ of pyrope compositions of garnet in this study are classified as Group A eclogitic garnets (Coleman et al., 1965), which usually occur in layers in ultramafic rocks. Most of the feldspar inclusions in these rubies and purple sapphires are plagioclase, with the exception of one alkali feldspar inclusion with anorthoclase composition. Anorthoclase and sanidine crystals are intergrown with sapphire in xenoliths from alkali basalt that suggest crystallization from intermediate magmas in the lower crust/upper mantle (Upton et al., 1983; Aspen et al., 
1990; Coenraads et al., 1990). The compositions of the pyroxene, garnet, plagioclase, and spinel inclusions support mafic granulites as the possible source of ruby and purple sapphire, as reported by Sutthirat et al. (2018) and Promprated et al. (2003). Nepheline, the most common of the feldspathoid minerals, occurs in alkaline rocks (Deer et al., 2013). Sapphirine inclusions support the equilibrium of garnet, corundum, spinel, and sapphirine. Anorthoclase, sillimanite, and silicate melt inclusions offer further evidence that the host formed from partial melting at high temperature (Palke et al., 2018). The exogenous mineral inclusions such as anhydrite, sulfide, and quartz observed in Bo Welu ruby and purple sapphire may be due to submarine volcanism prior to subduction, when these shallow crustal materials moved downward and mixed with mafic materials at the mantle. The chemical composition data of the mineral inclusions implies that these samples crystallized at high temperature through partial melting of hybrid mantle material affected by ancient subduction. Therefore, their original source is confirmed to be mafic granulites characterized by high pressure and very high temperature.

The inclusions in Bo Welu blue sapphire consist of alkaline-related minerals that crystallized from alkaline felsic magma, such as alkali feldspar, monazite, columbite, and zircon. The feldspar inclusions found in this study were mainly Na-rich alkali feldspar (albite), with an unusual sanidine. The low Ca content of the feldspars indicates that the source rock was felsic. Sanidine suggests that the crystallization of blue sapphire may be related to high-temperature metamorphism. Saminpanya and Sutherland (2011) proposed that sanidine inclusions in Den Chai sapphire may have crystallized at a minimum temperature of about $1000^{\circ} \mathrm{C}$. Monazite inclusions in sapphires suggest that crystallization of basaltic sapphire is related to highly evolved melt (Krzemnicki et al., 1996; Intasopa et al., 1998; Singbamroong and Thanasuthipitak, 2004; Khamloet et al., 2014). Columbite generally occurs in various granitic rocks (Guo et al., 1996). Chemical characteristics of zircons (e.g., high $\mathrm{Hf}, \mathrm{Y}$, and rare earth elements) have been found in sapphires from basaltic terrains elsewhere, possible evidence that the host sapphire crystallized from alkaline and highly evolved source material under conditions unrelated to the associated basaltic magma (e.g., Coenraads et al., 1990, 1995; Guo et al., 1996; Sutherland et al., 2002; Khamloet et al., 2014). In addition, the exogenous inclusions in sapphire such as sulfides indicate starting materials related to subduc- tion and hybridization within the deep crust and later partial melting of initial magma-related sapphire formation. The occurrence of mineral inclusions in Bo Welu blue sapphire strongly supports crystallization from highly evolved alkali-rich, silica-poor magma (syenite) (e.g., Coenraads et al., 1990; Ding, 1991; Coenraads et al., 1995; Guo et al., 1996; Sutherland et al., 2002; Khamloet et al., 2014).

The Chanthaburi-Trat placer rubies and sapphires should have a somewhat different crystallization environment, as suggested by previous research (e.g., Yui et al., 2006; Saminpanya and Sutherland, 2011). The ruby formed at greater depths (the upper mantle) than the sapphire (crust/upper mantle). However, ruby and sapphire have been found together in the central Chanthaburi-Trat deposits, including Bo Welu, which should have geological linkage between deeper ruby formation, narrower sapphire formation, and the deepest basaltic magma source prior to basaltic magma that has risen and then brought ruby, sapphire, and other associated assemblages up to the surface via volcanic eruption. More detailed investigation and further discussion of this complex geological process can be carried out on the basis of this study.

\section{CONCLUSIONS}

Although ruby and sapphire production from Bo Welu has declined since the 1970s, a few artisanal miners are still supplying the market. These stones are in high demand for their colors: purplish red ruby and green-blue sapphire. They are clearly characterized as basaltic-type corundum, based on their primary corroded surface.

Various types of mineral inclusions have been identified in this study, and their chemical composition can be used to interpret the conditions in which ruby and sapphire crystallized prior to being transported to the surface by basaltic magma. Many of these inclusions are common; for others, such as sillimanite, nepheline, quartz, alkali feldspar, spinel, and anhydrite for ruby and purple sapphire, and sulfide for blue sapphire, this is a newly reported occurrence in Thai corundum. These inclusions can be used to support origin determination. The original deep-seated formations of Thai ruby and sapphire as well as transportation by basaltic volcanism are actually complicated geological processes that must take place under very specific conditions. Therefore, these rubies and sapphires should be valued not only for their rarity and quality but also their unique geological history. 
ABOUT THE AUTHORS

Ms. Promwongnan is a master's degree student in geology at Chulalongkorn University and a gemologist at the Gem Testing Laboratory of the Gem and Jewelry Institute of Thailand (GIT). Dr. Sutthirat (chakkaphan.s@chula.ac.th) is a professor of earth sciences at the Geology Department, Faculty of Science, Chulalongkorn University. He has also served as an academic advisor to the GIT's Gem Testing Laboratory.

\section{ACKNOWLEDGMENTS}

This research is financially supported by the 90th Anniversary of
Chulalongkorn University, Rachadapisek Sompote Fund of Chulalongkorn University. All analyses were carried out at the Geology Department, Faculty of Science, Chulalongkorn University and the GIT's Gem Testing Laboratory. The first author would like to thank Duangkamol Jiambutr (GIT director), Wilawan Atichat, Visut Pisutha-Arnond, Thanong Leelawattanasuk, and Sakonvan Chawchai for their suggestions and encouragement. Special thanks are also be given to Sopit Poompeang for assistance with sample preparation and EPMA analysis. Finally, we must thank all the technical reviewers for handling the manuscript with very constructive comments and kind support.

\section{REFERENCES}

Aspen P., Upton B.G.J., Dickin A.P. (1990) Anorthoclase, sanidine and associated megacrysts in Scottish alkali basalts: high-pressure syenitic debris from upper mantle sources? European Journal of Mineralogy, Vol. 2, No. 4, pp. 503-517, http://dx.doi.org/ $10.1127 / \mathrm{ejm} / 2 / 4 / 0503$

Barr S., Cooper M. (2013) Late Cenozoic basalt and gabbro in the subsurface in the Phetchabun Basin, Thailand: Implications for the Southeast Asian Volcanic Province. Journal of Asian Earth Sciences, Vol. 76, pp. 169-184, http://dx.doi.org/10.1016/j. jseaes.2013.01.013

Černý P., Ercit T.S. (1985) Some recent advances in the mineralogy and geochemistry of $\mathrm{Nb}$ and $\mathrm{Ta}$ in rare-element granitic pegmatites. Bulletin de Mineralogie, Vol. 108, pp. 499-532, http://dx.doi.org/10.3406/bulmi.1985.7846

Coenraads R.R. (1992) Surface features on natural rubies and sapphires derived from volcanic provinces. Journal of Gemmology, Vol. 23, No. 3, pp. 151-160.

Coenraads R.R., Sutherland F.L., Kinny P.D. (1990) The origin of sapphires: $\mathrm{U}-\mathrm{Pb}$ dating of zircon inclusions sheds new light. Mineralogical Magazine, Vol. 54, No. 374, pp. 113-122, http://dx.doi.org/10.1180/minmag.1990.054.374.13

Coenraads R.R., Vichit P., Sutherland F.L. (1995) An unusual sapphire-zircon-magnetite xenolith from the Chanthaburi Gem Province, Thailand. Mineralogical Magazine, Vol. 59, No. 396, pp. 465-479, http://dx.doi.org/10.1180/minmag.1995.059.396. 08

Coleman R.G., Lee D.E., Beatty L.B., Brannock W.W. (1965) Eclogites and eclogites: their differences and similarities. Geological Society of America Bulletin, Vol. 76, No. 5, pp. 483-508, http://dx.doi.org/10.1130/0016-7606/1965/76\%5B483: EAETDA\%5D2.0.CO;2

Deer W.A., Howie R.A., Zussman J. (2013) An Introduction to the Rock-forming Minerals, 3rd ed. Longman, Essex, UK, 696 pp.

Ding Z. (1991) Mineralogical studies on Shandong sapphires (unpublished). MSc thesis, Academia Sinica Inst Geochem, Guiyang, China.

Droop G. (1987) A general equation for estimating $\mathrm{Fe}^{3+}$ concentrations in ferromagnesian silicates and oxides from microprobe analyses, using stoichiometric criteria. Mineralogical Magazine, Vol. 51, No. 361, pp. 431-435, http://dx.doi.org/10.1180/ minmag.1987.051.361.10

Gübelin E.J. (1940) Differences between Burma and Siam rubies. Ge G, Vol. 3, No. 5, pp. 69-72.

Gübelin E.J. (1971) New analytical results of the inclusions in Siam rubies. Journal of Australian Gemmology, Vol. 12, No. 7, pp. 242-252.

Gübelin E.J., Koivula J.I. (1986) Photoatlas of Inclusions in Gemstones, Volume 1. ABC Edition, Zurich, $532 \mathrm{pp}$.

Guo J., Griffin W.L., O'Reilly S.Y. (1994) A cobalt-rich spinel in- clusion in a sapphire from Bo Ploi, Thailand. Mineralogical Magazine, Vol. 58, No. 391, pp. 247-258, http://dx.doi.org/ 10.1180/minmag.1994.058.391.07

Guo J., O'Reilly S.Y., Griffin W.L. (1996) Corundum from basaltic terrains: a mineral inclusion approach to the enigma. Contributions to Mineralogy and Petrology, Vol. 122, No. 4, pp. 368386, http://dx.doi.org/10.1007/s004100050134

Hughes R.W. (1997) Ruby «) Sapphire. RWH Publishing, Boulder, Colorado.

Intasopa S., Atichat W., Pisutha-Arnond V. (1998) Inclusions in corundum: A new approach to the definition of standards for origin determination. Science and Technology for Gem and Jewelry Industry Thailand Research Fund [in Thai].

Intasopa S., Atichat W., Pisutha-Arnond V., Sriprasert B., Narudeesombat N., Phuttarat T. (1999) Inclusions in Chanthaburi - Trat corundums: A clue to their genesis, in Thai. Proceedings of the Symposium on Mineral, Energy, and Water Resources of Thailand: Towards the Year 2000, October 28-29, 1999, Bangkok, Thailand, pp. 471-484.

Khamloet P., Pisutha-Arnond V., Sutthirat C. (2014) Mineral inclusions in sapphire from the basalt-related deposit in Bo Phloi, Kanchanaburi, Western Thailand: indication of their genesis. Russian Geology and Geophysics, Vol. 55, No. 9, pp. $1087-$ 1102, http://dx.doi.org/10.1016/j.rgg.2014.08.004

Koivula J.I., Fryer C.W. (1987) Sapphirine (not sapphire) in a ruby from Bo Rai, Thailand. Journal of Gemmology, Vol. 20, No. 6, pp. 369-370.

Krzemnicki M.S., Hänni H.A., Guggenheim R., Mathys D. (1996) Investigations on sapphires from an alkali basalt, South West Rwanda. Journal of Gemmology, Vol. 25, No. 2, pp. 90-106.

Palke A.C., Wong J., Verdel C., Ávila J.N. (2018) A common origin for Thai/Cambodian rubies and blue and violet sapphires from Yogo Gulch, Montana, U.S.A? American Mineralogist, Vol. 103 , No. 3, pp. 469-479, http://dx.doi.org/10.2138/am-20186164

Pattamalai K. (2015) Chanthaburi-Trat corundum deposits, Eastern Thailand. Proceedings of The 3rd Lao-Thai Technical Conference, July 7-8, 2015, pp. 219-229.

Promprated P., Taylor L.A., Neal C.R. (2003) Petrochemistry of mafic granulite xenoliths from the Chantaburi basaltic field: implications for the nature of the lower crust beneath Thailand. International Geology Review, Vol. 45, No. 5, pp. 383406, http://dx.doi.org/10.2747/0020-6814.45.5.383

Promwongnan S., Sutthirat C. (2019) An update on mineral inclusions and their composition in ruby from the Bo Rai gem field in Trat Province, eastern Thailand. Journal of Gemmology, Vol. 36, No. 7, pp. 634-645, http://doi.org/10.15506/JoG.2019. 36.7.634

Saeseaw S., Sangsawong S., Vertriest W., Atikarnsakul U., Liliane 
R.-F.V., Khowpong C., Weeramonkhonlert V. (2017) A study of sapphire from Chanthaburi, Thailand and its gemological characteristics. GIA Research News, www.gia.edu/gia-newsresearch/sapphire-chanthaburi-thailand-gemological-characteristics

Saminpanya S., Sutherland F.L. (2011) Different origins of Thai area sapphire and ruby, derived from mineral inclusions and co-existing minerals. European Journal of Mineralogy, Vol. 23, No. 4, pp. 683-694.

Sangsawong S., Vertriest W., Saeseaw S., Pardieu V., Muyal J., Khowpong C., Atikarnsakul U., Weeramonkhonlert V. (2017) A study of rubies from Cambodia \& Thailand. GIA Research News, https://www.gia.edu/gia-news-research/study-rubiescambodia-thailand

Singbamroong S., Thanasuthipitak T. (2004) Study of solid mineral inclusions in sapphires from Ban Huai Sai area, Laos, by Raman spectroscopy. Chiang Mai Journal of Science, Vol. 31, No. 3, pp. 251-263.

Sutherland F.L., Hoskin P.W., Fanning C.M., Coenraads R.R. (1998a) Models of corundum origin from alkali basaltic terrains: a reappraisal. Mineralogy and Petrology, Vol. 133, No. 4, pp. 356-372.

Sutherland F.L., Schwarz D., Jobbins E.A., Coenraads R.R., Webb G. (1998b) Distinctive gem corundum suits from discrete basalt fields: a comparative study of Barrington, Australia, and West Pailin, Cambodia, gemfields. Journal of Gemmology, Vol. 26, No. 2, pp. 65-85.

Sutherland F.L., Bosshart G., Fanning C.M., Hoskin P.W.O., Coenraads R.R. (2002) Sapphire crystallization, age and origin, Ban
Huai Sai Laos: age based on zircon inclusions. Journal of Asian Earth Sciences, Vol. 20, No. 7, pp. 841-849.

Sutherland F.L., Coenraads R.R., Schwarz D., Raynor L.R., Barron B.J., Webb G.B. (2003) Al-rich diopside in alluvial ruby and corundum-bearing xenoliths, Australian and SE Asian basalt fields. Mineralogical Magazine, Vol. 67, No. 4, pp. 717-732.

Sutthirat C., Saminpanya S., Droop G.T.R., Henderson C.M.B., Manning D.A.C. (2001) Clinopyroxene-corundum assemblages from alkali basalt and alluvium, eastern Thailand: constraints on the origin of Thai rubies. Mineralogical Magazine, Vol. 65, No. 2, pp. 277-295.

Sutthirat C., Hauzenberger C., Chualaowanich T., Assawincharoenkij T. (2018) Mantle and deep crustal xenoliths in basalts from the Bo Rai ruby deposit, Eastern Thailand: Original source of basaltic ruby. Journal of Asian Earth Sciences, Vol. 164, pp. 366-379.

Upton B.G.J., Aspen P., Chapman N.A. (1983) The upper mantle and deep crust beneath the British Isles: evidence from inclusions in volcanic rocks. Journal of the Geological Society of Thailand, Vol. 140, No. 1, pp. 105-121.

Vichit P. (1992) Gemstones in Thailand. In C. Piencharoen, Ed., Proceedings of a National Conference on Geologic Resources of Thailand-Potential for Future Development: 17-24 November 1992, Bangkok, Thailand. Department of Mineral Resources, Bangkok.

Yui T.-F., Wu C.-M., Limtrakun P., Sricharn W., Boonsoong A. (2006) Oxygen isotope studies on placer sapphire and ruby in the Chanthaburi-Trat alkali basaltic gemfield, Thailand. Lithos, Vol. 86, No. 3-4, pp. 197-211.

For online access to all issues of GEMS \& GEMOLOGY from 1934 to the present, visit: 